Citation: G. Gilardi, M. Pugliese, M.L. Gullino, A. Garibaldi (2020) Evaluation of different carbon sources for anaerobic soil disinfestation against Rhizoctonia solani on lettuce in controlled production systems. Phytopathologia Mediterranea 59(1): 77-96. doi: 10.14601/ Phyto-10911

Accepted: December 19, 2019

Published: April 30, 2020

Copyright: (c) 2020 G. Gilardi, M. Pugliese, M.L. Gullino, A. Garibaldi. This is an open access, peer-reviewed article published by Firenze University Press (http://www.fupress.com/pm) and distributed under the terms of the Creative Commons Attribution License, which permits unrestricted use, distribution, and reproduction in any medium, provided the original author and source are credited.

Data Availability Statement: All relevant data are within the paper and its Supporting Information files.

Competing Interests: The Author(s) declare(s) no conflict of interest. The Author Massimo Pugliese declares he has a financial interest (shareholder) in AgriNewTech, the company that provided the compost tested in this research.

Editor: Jean-Michel Savoie, INRA Villenave d'Ornon, France.

\section{Research Paper \\ Evaluation of different carbon sources for anaerobic soil disinfestation against Rhizoctonia solani on lettuce in controlled production systems}

\author{
Giovanna GILARDi ${ }^{1, \star}$, Massimo PUGLIESE ${ }^{1,2}$, Maria Lodovica GULLI- \\ $\mathrm{NO}^{1,2}$, ANGELO GARIBALDI ${ }^{1}$ \\ ${ }^{1}$ Centre for Innovation in the Agro-Environmental Sector, AGROINNOVA, University of \\ Torino, Largo Braccini 2, 10095 Grugliasco (TO), Italy \\ ${ }^{2}$ Department of Agricultural, Forest and Food Sciences (DISAFA), University of Torino, \\ Largo Braccini 2, 10095 Grugliasco (TO), Italy \\ *Corresponding author: giovanna.gilardi@unito.it
}

Summary. Effects of anaerobic soil disinfestation (ASD) on Rhizoctonia solani basal rot of lettuce were assessed considering: two soil types; different C-sources; different temperature regimes; two treatment durations; and two lettuce crop cycles, in the presence of a high disease incidence from artificial infestation with the pathogen. C-source, temperature, and incubation period, and their interaction, affected the efficacy of the ASD treatment for the lettuce- $R$. solani pathosystem, with differences depending on the soil type. Brassica carinata pellets, used as a C-source, reduced incidence of Rhizoctonia basal rot in the first crop cycle by 50 to $69 \%$ in a peat soil after 3 weeks of treatment at $21^{\circ} \mathrm{C}$, and by 52 to $60 \%$ after 3 weeks of treatment at 26 or $31^{\circ} \mathrm{C}$, compared to the inoculated and untreated experimental controls without anaerobic conditions. The best disease reduction was provided by $B$. carinata pellets applied, under anaerobic conditions, to peat soil (79\% efficacy) and a sandy loam soil ( $100 \%$ efficacy) kept at $31^{\circ} \mathrm{C}$ for 6 weeks. Generally, ASD based on B. carinata pellets provided greater disease reduction in the first crop cycle than the second, with the only exception being for results achieved in both soils incubated for 6 weeks at 21,26 and $31^{\circ} \mathrm{C}$. Wild rocket used as a C-source provided the greatest disease reduction $(78-83 \%)$ on plants grown in peat soil at the first crop cycle after 6 weeks of the ASD treatment at $31^{\circ} \mathrm{C}$, while wild rocket provided disease reduction of 29 and 50\% when mixed with the sandy-loam soil under the same conditions for 6 weeks. The efficacy of the ASD treatment with compost was improved in the second crop cycle, compared to the first, resulting in the greatest disease reduction (52 and 66\% efficacy) reached in the sandy-loam soil incubated for 3 weeks at $21^{\circ} \mathrm{C}$, and $63 \%$ efficacy in peat soil previously treated for 6 weeks at 26 and $31^{\circ} \mathrm{C}$. However, slight reductions in lettuce plant development was generally evident in the first crop cycle, which could be due to differences in efficacy of the tested ASD treatments and to phytotoxicity. The greatest yield from sandy loam soil was obtained for the $B$. carinata pellets and wild rocket at 26 and $31^{\circ} \mathrm{C}$ in the second cultivation cycle. Partial efficacy of ASD does not justify the adoption of this method against $R$. solani on lettuce under intensive crop systems. However, ASD based on Brassicaceae and compost as carbon source applied in a sandy-loam soil may be valuable for reducing $R$. solani incidence, at lower temperatures than those required for soil solarisation or biosolarisation.

Keywords. Lactuca sativa, pre-plant treatments, soil-borne pathogens, biological soil disinfestation. 


\section{INTRODUCTION}

Anaerobic soil disinfestation (ASD) is a method based on the anaerobic decomposition of organic matter, and this was developed as a possible alternative to the use of fumigants (Shennan et al., 2014). ASD is based on two systems, one developed in the Netherlands, as a biological soil disinfestation (Blok et al., 2000), and the other in Japan, as a soil reductive sterilisation process (Shinmura, 2004). This is applied in Japan, the Netherlands, the United States of America, and Argentina, to control of different pathogens and pests under practical conditions (Rosskopf et al., 2015; Shrestha et al., 2016; Shennan et al., 2018).

The ASD process is based on the addition of labile carbon sources to the soil to stimulate microbial growth and respiration, followed by irrigation to fill the water pore spaces, and covering the soil with plastic films, to reduce gas exchange. This then permits diffusion through the soil of by-products of decomposition, as well as reduced soil oxygen (Butler et al., 2012a, 2012b; Shennan et al., 2014). These treatments establish anaerobic conditions, as the aerobic microorganisms consume the remaining oxygen present in the soil, and the microbial communities shift to facultative and obligate anaerobes (Mazzola et al., 2007; Momma et al., 2010; Mowlick et al., 2012, 2013; Huang et al., 2016; Hewavitharana and Mazzola, 2016). After the anaerobic conditions have been maintained for a period, depending on soil temperature and the type of C-source used, the soil is uncovered to allow oxygen to return, stimulating the degradation of the remaining anaerobic decomposition by products (Shennan et al., 2014). ASD is effective against soil-borne pathogens and pests through different mechanisms, including production of organic acids via anaerobic decomposition of the added C, production of volatiles (Okazaki and Nose, 1986; Momma et al., 2006; Mazzola and Hewavitharana, 2014; Huang et al., 2016), and biocontrol activity of fungal and bacterial communities that grow during the process (Momma et al., 2010; Mazzola and Manici, 2012; Mowlick et al., 2012, 2013; Runia et al., 2012, 2014; Butler et al., 2014 a, 2014b).

Most studies and practical applications of ASD have dealt with crops such as asparagus, tomato, pepper, eggplant, cucumber, melon, spinach, strawberry, cut flowers and fruit trees (Blok et al., 2000; Mazzola et al., 2001; Goud et al., 2004; Messiha et al., 2007; Yossen et al., 2008; Lamers et al., 2010; Mazzola and Manici, 2012; Butler et al., 2012 a, 2012b; Mowilck et al., 2013; Hewavitharana and Mazzola, 2016; Serrano-Pérez et al., 2017; Shennan et al., 2014, 2018). The
C-sources used generally depends on the availability of inexpensive or waste materials in the different locations, and there is variability among countries. Among the tested C-sources, the most common have been: wheat bran (Yossen et al., 2008; Momma et al., 2010), rice bran (Shennan et al., 2010; Strauss and Kluepfel, 2015), ryegrass (Blok et al., 2000; Goud et al., 2004), molasses (Momma et al., 2010, 2013; Butler et al., 2012 a), diluted ethanol (Momma et al., 2010; Hewavitharana and Mazzola, 2013), green manure (Butler et al., 2012b; Mowilck et al., 2013; Hewavitharana et al., 2014; McCarty et al., 2014), composted broiler litter (Hewavitharana et al., 2014; Di Gioia et al., 2017), and residues of different cover crops. Amendment rates with these materials have varied between 3 to $90 \mathrm{tha}^{-1}$ (Shrestha et al., 2016).

ASD has not yet been practically applied in Italy, and there is still a need to adapt this soil disinfestation method to the cultural and environmental conditions of this country. The choice of effective, cheap and easily available C-sources is of particular importance. Although a number of studies have reported effects of ASD on soilborne pathogens of many crops, no studies have been carried out on leafy vegetables. These represent an intensive production system in many countries, and are particularly important in Italy, both in open fields and protected systems (Gullino et al., 2019). There is no standardised ASD method to determine the best combination for soil-borne pathogen control, and several factors, such as the $\mathrm{C}$-source, the rate of application, the treatment duration, the temperature under the plastic covers or the type of soil, can influence the effectiveness of this disease management method.

Rhizoctonia solani (Kühn), the soil-borne fungus that causes basal rot of a broad range of hosts, is one of the most important pathogens affecting lettuce production in Italy. This pathogen is also important in most countries where lettuce is grown (Blancard et al., 2003; Barrière et al., 2014; Gullino et al., 2019).

The present study aimed to evaluate effects of ASD on the $R$. solani-lettuce pathosystem, under controlled conditions, and considering several factors. These included: i) different carbon sources; ii) different temperature regimes; and iii) two treatment durations. The effects on disease incidence and lettuce fresh weight were evaluated in two soil types considering two crop cycles planted in an ASD-treated soil, and in reference experimental controls with or without anaerobiotic conditions. Results obtained have been compared with those from tolclofos-methyl, the available fungicide for control of $R$. solani basal rot of lettuce. 


\section{MATERIALS AND METHODS}

\section{Experimental layout}

Two trials were carried out at the Agroinnova Centre of Competence of the University of Torino, Grugliasco, under controlled conditions. ASD treatments were applied in four growth chambers and in two greenhouse compartments (64 $\mathrm{m}^{2}$ each), to test the treatment efficacies against $R$. solani. Different carbon sources, including compost (Comp), Diplotaxis tenuifolia green manure (WR) and Brassica carinata pellets (BCp), and two types of soil (peat and sandy-loam) were used, at different temperatures $\left(21,26\right.$ or $31^{\circ} \mathrm{C}$ ) and two durations (3 and 6 weeks) as the main experiment factors. The ASD treatments were carried out in plastic containers (capacity, 40 $\mathrm{L}$; dimensions $50 \times 40 \times 20 \mathrm{~cm})$, soil surface area $(2,000$ $\left.\mathrm{cm}^{2}\right)$ using two types of soil: i) a mixture (50:50 v:v) of a sandy loam soil (sand : silt : loam, $68.16: 10.7: 21.1 ; \mathrm{pH}$ 7.9; organic matter $0.94 \%$ ), and perlite (Perlite Italiana Agrilit 3); ii) a peat substrate (Tecno 2, 70\% white peat and 30\% clay; $\mathrm{pH} 6.1-6.5 ; \mathrm{N} 110-190 \mathrm{mg} \mathrm{L}^{-1} ; \mathrm{P}_{2} \mathrm{O}_{5} 140-$ $230 \mathrm{mg} \mathrm{L}^{-1} ; \mathrm{K}_{2} \mathrm{O}$ 170-280 $\mathrm{mg} \mathrm{L}^{-1}$, Turco Silvestro terricci). The plastic containers were filled with $30 \mathrm{~L}$ of the treated or untreated soil distributed in a layer of $15 \mathrm{~cm}$ deep.

At the end of each ASD incubation period carried out in the growth chambers, the treated and untreated soil from each container was redistributed into four 12 $\mathrm{L}$ capacity plastic pots and kept in two compartments in a greenhouse at temperatures ranging from 27 to $30^{\circ} \mathrm{C}$, and relative humidity $(\mathrm{RH})$ of $70-80 \%$. The pots were arranged on benches in a completely randomized block design with one pot serving as a replicate, using four replicates per treatment. The experimental design of the trials is illustrated in Figure 1.

ASD treatment simulated under growth chamber conditions, and measurements

The ASD treatments started immediately, under controlled conditions in the growth chambers, after addition of the selected C-sources to the soil, and application of irrigation water at $17.0 \mathrm{~L}$ per container for the peat soil, or 7.0 L per container for the sandy loam soil. These irrigation rates were estimated by adding excess water to the respective soils and then allowing water to drain for $24 \mathrm{~h}$, to simulate saturation conditions. The soil was then covered with standard polyethylene (PE) sheets $(50$ $\mu \mathrm{m}$ thick), immediately after the application of the different carbon sources and water, and the containers were moved to the growth chambers to start the ASD treatments at different temperatures.
The soils were incubated for 3 or 6 weeks at constant temperatures of $21^{\circ} \mathrm{C}$ (Trials 1 and 2), and at 26 (Trial 1) or $31^{\circ} \mathrm{C}$ (Trial 2) (Figure 1). These temperatures were selected to simulate typical soil conditions in the Mediterranean area during spring and summer (Tamietti and Garibaldi, 1987). The soil temperatures were monitored using a sensor data logging system (Digital Data Logger EM50; Decagon Devices). Redox potential values were taken manually each day ( $5 \mathrm{~d}$ per week, for 3 or 6 weeks), using an ORP/temp pen style meter (VWR International) at three positions in each container. The temperature and redox sensors were placed at the centre of each container at a depth of $10 \mathrm{~cm}$.

The ORP values of the soil redox potential, expressed in $\mathrm{mV}$, were converted to $\mathrm{Eh} \mathrm{mV}$, related to the redox potential of a standard hydrogen electrode, by adding $200 \mathrm{mV}$ (Fiedler et al., 2007). To calculate the cumulative soil anaerobic conditions, the absolute values of the difference between each redox value and the critical redox potential (CEh), calculated using the formula: $595 \mathrm{mV}-(60 \mathrm{mV} \times$ soil $\mathrm{pH}$ measured at the end of the ASD treatment), were summed for redox values below $\mathrm{CEh}$. Since the recorded data were the average daily redox potential values, the values obtained were multiplied by $24 \mathrm{~h}$ and converted into hourly units (mVh), then summed for each day. The cumulative soil anaerobic conditions $(\mathrm{mVh})$ over the 3 or 6 weeks of the ASD treatments was then obtained (Rabenhorst and Castenson, 2005; Butler et al., 2012 a; b).

\section{Artificial infestation with the pathogen}

One isolate (code AG2-L) of R. solani, obtained from affected lettuce, was used. The isolate was grown on PDA plates for $10 \mathrm{~d}$. Flasks (1 L capacity), containing $300 \mathrm{~g}$ of sterilised wheat kernels, were inoculated with 5 $\mathrm{mm}$ diam. agar disks from $R$. solani PDA colonies, and were then maintained for $20 \mathrm{~d}$ at $23^{\circ} \mathrm{C}$. Soils were infested with the pathogen by mixing $0.5 \mathrm{~g} \mathrm{~L}^{-1}$ of the infested kernels, immediately before commencing the ASD treatments, using a total of $15 \mathrm{~g}$ of inoculum per container. The same amount of non-infested kernels was used for the non-inoculated experimental controls.

\section{Carbon sources}

Four carbon sources, selected among a number of different sources used in preliminary trials, were tested, and these are reported hereafter at selected field doses selected, according to the recommendations by Butler et al. (2014b) for moderate soil temperature: 


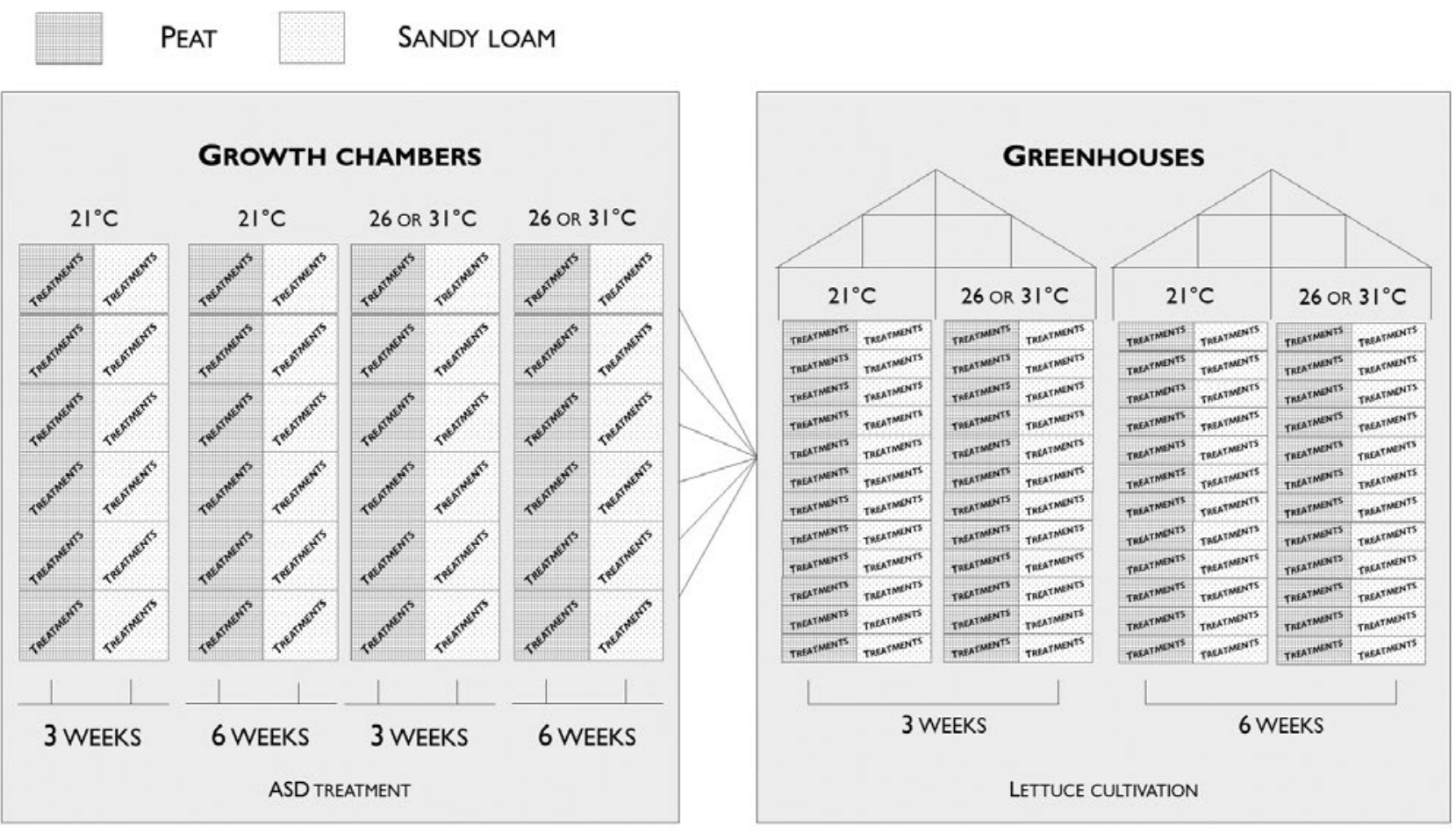

Figure 1. Experimental protocols utilized to assess the effect of anaerobic soil disinfestation (ASD) for the control of Rhizoctonia solani on lettuce. Assays were conducted in four growth chambers and in two greenhouse compartments during two trials carried out in peat and sandy-loam soils.

i) WR, wild rocket (Diplotaxis tenuifolia, C/N 6.9) applied as a green manure crop, at $10 \mathrm{~g} \mathrm{~L}^{-1}$, equivalent to $15 \mathrm{t} \mathrm{ha}^{-1} 15 \mathrm{~cm}$ depth, or at $20 \mathrm{~g} \mathrm{~L}^{-1}$, equivalent to $30 \mathrm{tha}^{-1}$;

ii) BCp, Brassica carinata defatted pellet ('Biofence': $\mathrm{C} / \mathrm{N} 10.96$, organic $\mathrm{N} 6 \%, \mathrm{P} 2.2 \%, \mathrm{~K} 2 \%$, organic C 52\%: Triumph), mixed into the soil at $10 \mathrm{~g} \mathrm{~L}^{-1}$, equivalent to $15 \mathrm{t} \mathrm{ha}^{-1} 15 \mathrm{~cm}$ depth;

iii) Comp, compost prepared from green wastes in a dynamic industrial treatment system, and passed through a $20 \mathrm{~mm}$ sieve (Ant's Compost V, lot number N-2015, AgriNewTech: C/N 16.5, pH 6.58; total N $0.971 \%$; total C $16 \% ; \mathrm{P}_{2} \mathrm{O}_{5} 0.57 \% ; \mathrm{K}_{2} \mathrm{O} 1.35 \%$ ), mixed into the soil at $10 \mathrm{~g} \mathrm{~L}^{-1}$, equivalent to $15 \mathrm{tha}^{-1} 15 \mathrm{~cm}$ depth.

Diplotaxis tenuifolia, 'Grazia', was sown at 1-1.2 $\mathrm{g} \mathrm{m}^{-2}$ in plastic pots filled with sterile blonde sphagnum peat, to produce wild rocket green manure. The pots were kept in a greenhouse at $24-26^{\circ} \mathrm{C}$ for $40-50 \mathrm{~d}(50 \%$ flowering). The plants were harvested, weighed, and cut into 1 to $3 \mathrm{~cm}$ pieces and incorporated into the soil, immediately before commencing the ASD treatment.

\section{Experimental control treatments}

Tolclofos-methyl TM (Rizolex, BASF Crop Protection, $50 \%$ a.i.) was used at $2 \mathrm{~g} \mathrm{~m}^{-2}$ in both trials as a fun- gicide experimental control, because this compound is effective for management of basal rot of lettuce, caused by $R$. solani (Sneh et al., 1996). In Trial 2, the commercial formulation 'Biofence' based on Brassica carinata defatted pellets was applied without anaerobic conditions, at the same rate used for the ASD treatment, as reference control (BCp-No anaerobic control).

Two inoculated untreated controls, without any carbon source incorporated into the soil but with differences in the volume of water used, were prepared:

i) a standard untreated control (INT-Standard control): irrigated to field soil capacity;

ii) an INT-Anaerobic control: irrigated to exceed field capacity, to provide reducing conditions.

In the inoculated and non-inoculated untreated controls (INT and NINT) as well as for the BCp-No anaerobic control, water equivalent to the moisture capacity of the peat soil ( $8.0 \mathrm{~L}$ per container) and sandy loam soil (5.0 L per container) was used. Two non-inoculated control pots, which received sterile wheat kernels, under an anaerobic condition (NINT- Anaerobic control) and under a standard condition (NINT-Standard control), were also included.

Both INT and NINT standard and anaerobic controls as well TM and BCp- No anaerobic control were 
each covered with a transparent polyethylene film (50 $\mu \mathrm{m}$ thick) for the 3 or 6 weeks time treatments.

\section{Effects of ASD treatment in greenhouse trials}

At the end of the ASD treatments carried out in growth chambers, the soil was transferred into $12 \mathrm{~L}$ capacity pots and was then aerated for 1 week before planting lettuce 'Elisa' (20 plants per pot). Lettuce seedlings were transplanted 20 to 25 day after sowing into the treated and untreated soil in two subsequent crop cycles.

Fertilizer equivalent to $20 \mathrm{~kg} \mathrm{ha}^{-1}$ (N 18; P 18; K 18 $+2 \mathrm{MgO}$, Osmoform) was applied to the soil surfaces at the end of the first cycle in both trials.

Disease incidence (percent dead plants) was evaluated each week by counting the collapsed and dead plants that showed brown, sunken lesions and rotting at the base of the crowns. Dead plants were removed and randomly selected to perform pathogen isolation. The plants were removed from the soil at the end of each cropping cycle in both trials, at the full maturity stage, to evaluate the final Rhizoctonia basal rot incidence, and the fresh weights of all healthy plants were determined.

\section{Data analyses}

The experiments were carried out and analysed as two independent trials, at the end of the first and second crop cycles (Tables 1, 2 and 3). Data of mean accumulated soil anaerobic conditions values were subjected to analysis of variance (ANOVA) in the SPSS software, with means separation based Tukey's test $(P \leq 0.05)$. Disease inci-

Table 1. List and dates of the different operations carried out in greenhouse experiments.

\begin{tabular}{|c|c|c|}
\hline Operations & Trial 1 & Trial 2 \\
\hline $\begin{array}{l}\text { Artificial soil infestation with Rhizoctonia } \\
\text { solani }\end{array}$ & $17 / 01 / 16$ & $31 / 05 / 16$ \\
\hline $\begin{array}{l}\text { Start of the ASD treatments lasting } 6 \\
\text { weeks }\end{array}$ & $18 / 01 / 16$ & $1 / 06 / 16$ \\
\hline $\begin{array}{l}\text { Start of the ASD treatments lasting } 3 \\
\text { weeks }\end{array}$ & $8 / 02 / 16$ & $22 / 06 / 16$ \\
\hline Lettuce transplant (Cycle I) & $11 / 03 / 16$ & $20 / 07 / 16$ \\
\hline Disease assessments & $4 / 04 / 16$ & $10 / 08 / 16$ \\
\hline Lettuce fresh weights determined (Cycle I) & $4 / 04 / 16$ & $10 / 08 / 16$ \\
\hline Lettuce transplant (Cycle II) & $8 / 04 / 16$ & $11 / 08 / 16$ \\
\hline Disease assessments & $10 / 5 / 16$ & $20 / 09 / 16$ \\
\hline $\begin{array}{l}\text { Lettuce fresh weights determined (end of } \\
\text { Cycles I and II) }\end{array}$ & $10 / 05 / 16$ & $20 / 09 / 16$ \\
\hline
\end{tabular}

dence $(D I)$ and fresh weights $(F W)$ of the healthy lettuce plants were subjected to ANOVA using the univariate procedure in the SPSS software 25.0 statistical package. Levene's test was used to test the homogeneity of variances. The DI data, expressed as a percentage of affected plants, were arc-sin-transformed to stabilise the variances and normalise their distribution. The effect of each carbon source, temperature, ASD duration (3 or 6 weeks) for two types of soil (peat or sandy-loam), and their interactions for both crop cycles, were evaluated as main factors. When the effects of the tested factors were significant $(P$ $\leq 0.05$ ) and interactions were observed among the considered factors (Table 3), one-way ANOVA was carried out to evaluate the combined effects of the involved factors on the percent disease incidence and plant fresh weights. The means were separated using Tukey's test $(P \leq 0.05)$ when the multiple comparisons of the considered factors were shown to be significantly related.

\section{RESULTS}

\section{Impacts of the soil treatments on soil parameters}

On the basis of the oxidation-reduction potential (ORP), the reduced conditions in the ASD treatments of the peat and sandy-loam soils were generally reached within 4 to $5 \mathrm{~d}$ for all the tested temperatures. The lowest cumulative soil anaerobic conditions indicated by the redox potential were observed in the no $\mathrm{C}$ source control (INT-Standard control) in the sandy-loam soil at $21^{\circ} \mathrm{C}$ and $26^{\circ} \mathrm{C}$ for both 3 and 6 weeks, and in the peat soil at $21^{\circ} \mathrm{C}$ for 3 weeks, while increased values were observed in the INT- control under anaerobic conditions for 6 weeks at $31^{\circ} \mathrm{C}(85,766 \mathrm{mVh})$ (Table 2$)$. The greatest levels of cumulative anaerobic conditions were observed in the peat soil treated with WR at $15 \mathrm{~g} \mathrm{ha}^{-1}$ for 6 weeks at $26^{\circ} \mathrm{C}$ in Trial $1(211,935 \mathrm{mVh})$, and at $30^{\circ} \mathrm{C}$ for 6 weeks in Trial $2\left(180,162 \mathrm{mVh}\right.$ at $15 \mathrm{t} \mathrm{ha}^{-1}$ and $184,683 \mathrm{mVh}$ at $\left.30 \mathrm{t} \mathrm{ha}^{-1}\right)$. The ASD based-WR treatment at $15 \mathrm{ha}^{-1}$ in the sandy-loam soil at $31^{\circ} \mathrm{C}$ (trial 2) provided cumulative anaerobic conditions of $82,788 \mathrm{mVh}$, and at $30 \mathrm{tha}^{-1}$, $119,066 \mathrm{mVh}$. At $21^{\circ} \mathrm{C}$ cumulative anaerobic conditions were $43,947 \mathrm{mVh}$ from $15 \mathrm{tha}^{-1}$ and $72,440 \mathrm{mVh}$ from $30 \mathrm{t} \mathrm{ha}^{-1}$. The cumulative anaerobic conditions calculated for 6 weeks from the ASD based-BCp treatment were different among the trials, and the temperature conditions tested (from 106,054 to $176,736 \mathrm{mVh}$ in the peat soil and from 86,950 to $112,968 \mathrm{mVh}$ in the sandy-loam soil), with significantly lower $\mathrm{mVh}$ values for 3 weeks of ASD duration. Three weeks of ASD based-Comp in the peat soil at $21^{\circ} \mathrm{C}$ provided cumulative anaerobic conditions of the soil between 1,206 and $4,803 \mathrm{mVh}$ and of 
Table 2. Average soil $\mathrm{pH}$ and the mean cumulative soil redox potential $(\mathrm{mVh})$ indicating anaerobic conditions, for the peat and sandy-loam soils after different soil treatments. Standard errors are indicated.

\begin{tabular}{|c|c|c|c|c|c|c|c|c|c|c|c|c|c|c|}
\hline \multirow{3}{*}{$\begin{array}{l}\text { Carbon source } \\
\text { dosage }\end{array}$} & \multirow{3}{*}{ ASD } & \multirow{3}{*}{$\begin{array}{c}\text { Treatment } \\
\text { duration } \\
\text { (weeks) }\end{array}$} & \multicolumn{6}{|c|}{ Trial 1} & \multicolumn{6}{|c|}{ Trial 2} \\
\hline & & & \multicolumn{3}{|c|}{$21^{\circ} \mathrm{C}$} & \multicolumn{3}{|c|}{$26^{\circ} \mathrm{C}$} & \multicolumn{3}{|c|}{$21^{\circ} \mathrm{C}$} & \multicolumn{3}{|c|}{$31^{\circ} \mathrm{C}$} \\
\hline & & & $\mathrm{pH}$ & $\mathrm{mVh}$ & & $\mathrm{pH}$ & $\mathrm{mVh}$ & & $\mathrm{pH}$ & $\mathrm{mVh}$ & & $\mathrm{pH}$ & $\mathrm{mVh}$ & \\
\hline \multicolumn{15}{|l|}{ Peat soil } \\
\hline Comp, $15 \mathrm{t} \mathrm{ha}^{-1}$ & + & 3 & 6.6 & $1,206 \pm 603$ & $b^{c}$ & 6.7 & $12,532 \pm 889$ & de & 6.6 & $4,803 \pm 109$ & e & 6.6 & $13,667 \pm 837$ & de \\
\hline BCp, $15 \mathrm{t} \mathrm{ha}^{-1}$ & + & 3 & 7.1 & $41,235 \pm 3,405$ & $5 \mathrm{ab}$ & 7.2 & $34,666 \pm 1,987$ & $\mathrm{~cd}$ & 6.8 & $27,708 \pm 970$ & c & 6.9 & $50,409 \pm 1,705$ & $c$ \\
\hline WR, $15 \mathrm{t} \mathrm{ha}^{-1}$ & + & 3 & 7.0 & $25,330 \pm 7,491$ & $1 \mathrm{ab}$ & 7.0 & $33,646 \pm 6,014$ & $\mathrm{~cd}$ & 6.4 & $30,916 \pm 371$ & c & 6.6 & $30,696 \pm 1,242$ & $\mathrm{~cd}$ \\
\hline $\mathrm{WR}, 30 \mathrm{tha}^{-1}$ & + & 3 & $-b$ & - & & - & - & - & 6.3 & $31,373 \pm 1,028$ & $c$ & 6.7 & $47,706 \pm 3,148$ & $c$ \\
\hline $\mathrm{TM}, 2 \mathrm{gm}^{-2}$ & - & 3 & 6.6 & $0 \pm 0$ & $\mathrm{~b}$ & 6.8 & $0 \pm 0$ & e & 6.8 & $0 \pm 0$ & $\mathrm{e}$ & 6.8 & $0 \pm 0$ & $\mathrm{e}$ \\
\hline $\begin{array}{l}\text { BCp, } 15 \mathrm{t} \mathrm{ha}^{-1}-\mathrm{No} \\
\text { anaerobic control }\end{array}$ & - & 3 & - & - & & - & - & - & 6.8 & $0 \pm 0$ & e & 6.9 & $0 \pm 0$ & e \\
\hline INT- Anaerobic control & + & 3 & 6.8 & $650 \pm 620$ & $\mathrm{~b}$ & 6.6 & $4,811 \pm 1,941$ & $\mathrm{e}$ & 6.5 & $1685 \pm 822$ & e & 6.5 & $5,267 \pm 382$ & e \\
\hline INT- Standard control & - & 3 & 6.4 & $0 \pm 0$ & $\mathrm{~b}$ & 5.9 & $0 \pm 0$ & $\mathrm{e}$ & 6.7 & $0 \pm 0$ & e & 6.7 & $0 \pm 0$ & e \\
\hline C, $15 \mathrm{t} \mathrm{ha}^{-1}$ & + & 6 & 6.6 & $25,972 \pm 1,482$ & $2 \mathrm{ab}$ & 6.6 & $46,495 \pm 5,078$ & c & 6.6 & $18,205 \pm 1,267$ & $\mathrm{~d}$ & 6.5 & $95,550 \pm 4,349$ & $\mathrm{~b}$ \\
\hline BCp, $15 \mathrm{tha}^{-1}$ & + & 6 & 6.9 & $106,054 \pm 4,167$ & $7 \mathrm{a}$ & 7.1 & $145,895 \pm 1,0183$ & $3 \mathrm{~b}$ & 6.9 & $135,800 \pm 1,094$ & a & 6.7 & $176,736 \pm 1,094$ & $\mathrm{a}$ \\
\hline WR, $15 \mathrm{t} \mathrm{ha}^{-1}$ & + & 6 & 6.7 & $89,537 \pm 3,866$ & 6 a & 6.9 & $211,935 \pm 8,192$ & $\mathrm{a}$ & 6.6 & $92,639 \pm 5,773$ & $\mathrm{~b}$ & 6.6 & $180,162 \pm 4,002$ & $\mathrm{a}$ \\
\hline $\mathrm{WR}, 30 \mathrm{t} \mathrm{ha}^{-1}$ & + & 6 & - & - & & - & - & - & 6.7 & $101,487 \pm 2,181$ & $\mathrm{~b}$ & 6.5 & $184,683 \pm 7,705$ & $\mathrm{a}$ \\
\hline $\mathrm{TM}, 2 \mathrm{gm}^{-2}$ & - & 6 & 6.7 & $0 \pm 0$ & $\mathrm{~b}$ & 6.6 & $0 \pm 0$ & e & 6.8 & $0 \pm 0$ & $\mathrm{e}$ & 6.6 & $0 \pm 0$ & $\mathrm{e}$ \\
\hline $\begin{array}{l}\text { BCp, } 15 \mathrm{t} \mathrm{ha}^{-1}-\mathrm{No} \\
\text { anaerobic control }\end{array}$ & - & 6 & 6.6 & $0 \pm 0$ & $\mathrm{~b}$ & 6.7 & $0 \pm 0$ & e & 6.9 & $0 \pm 0$ & $\mathrm{e}$ & 6.7 & $0 \pm 0$ & $\mathrm{e}$ \\
\hline INT-Anaerobic control & + & 6 & 6.7 & $21,770 \pm 650$ & $\mathrm{ab}$ & 6.5 & $21,107 \pm 1,049$ & de & 6.5 & $23,949 \pm 1,562$ & $\mathrm{~cd}$ & 6.5 & $85,766 \pm 5,795$ & $\mathrm{~b}$ \\
\hline INT-Standard control & - & 6 & 6.3 & $0 \pm 0$ & $\mathrm{~b}$ & 6.1 & $0 \pm 0$ & e & 6.7 & $0 \pm 0$ & $\mathrm{e}$ & 6.6 & $0 \pm 0$ & $\mathrm{e}$ \\
\hline \multicolumn{15}{|l|}{ Sandy-loam soil } \\
\hline Comp, $15 \mathrm{t} \mathrm{ha}^{-1}$ & + & 3 & 7.8 & $1,017 \pm 709$ & $\mathrm{~b}$ & 7.3 & $2,606 \pm 1,598$ & $c$ & 7.8 & $1,914 \pm 475$ & $\mathrm{~d}$ & 7.9 & $2,805 \pm 1,426$ & $\mathrm{c}$ \\
\hline BCp, $15 \mathrm{t} \mathrm{ha}^{-1}$ & + & 3 & 7.4 & $10,822 \pm 4,962$ & $2 b$ & 7.3 & $23,579 \pm 2,335$ & $\mathrm{bc}$ & 7.5 & $11,315 \pm 2,507$ & $\mathrm{~cd}$ & 7.8 & $20,403 \pm 3,342$ & c \\
\hline WR, $15 \mathrm{t} \mathrm{ha}^{-1}$ & + & 3 & 7.0 & $10,980 \pm 1,371$ & $1 \mathrm{~b}$ & 7.3 & $22,110 \pm 971$ & $\mathrm{bc}$ & 7.7 & $10,937 \pm 4,042$ & $\mathrm{~cd}$ & 7.9 & $23,491 \pm 2,790$ & c \\
\hline $\mathrm{WR}, 30 \mathrm{t} \mathrm{ha}^{-1}$ & + & 3 & - & - & & & - & & 7.6 & $12,474 \pm 4,305$ & $\mathrm{~cd}$ & 7.6 & $25,470 \pm 2,221$ & c \\
\hline $\mathrm{TM}, 2 \mathrm{gm}^{-2}$ & - & 3 & 7.7 & $0 \pm 0$ & $\mathrm{~b}$ & 7.8 & $0 \pm 0$ & c & 7.1 & $0 \pm 0$ & $\mathrm{~d}$ & 7.8 & $0 \pm 0$ & c \\
\hline $\begin{array}{l}\text { BCp, } 15 \mathrm{t} \mathrm{ha}^{-1}-\mathrm{No} \\
\text { anaerobic control }\end{array}$ & - & 3 & - & - & & & - & & 7.8 & $0 \pm 0$ & $\mathrm{~d}$ & 7.9 & $0 \pm 0$ & c \\
\hline INT-Anaerobic control & + & 3 & 7.8 & $583 \pm 333$ & $\mathrm{~b}$ & 7.8 & $1,158 \pm 657$ & c & 7.9 & $36 \pm 36$ & $\mathrm{~d}$ & 7.9 & $1,272 \pm 756$ & c \\
\hline INT-Standard control & - & 3 & 7.8 & $0 \pm 0$ & $\mathrm{~b}$ & 7.8 & $0 \pm 0$ & c & 7.9 & $0 \pm 0$ & $\mathrm{~d}$ & 7.8 & $0 \pm 0$ & $c$ \\
\hline Comp, $15 \mathrm{t} \mathrm{ha}^{-1}$ & + & 6 & 7.1 & $8,632 \pm 1,495$ & $5 \mathrm{~b}$ & 7.1 & $15,516 \pm 4,228$ & c & 7.9 & $15,485 \pm 1,842$ & $\mathrm{~cd}$ & 7.5 & $31,817 \pm 1,593$ & bc \\
\hline BCp, $15 \mathrm{t} \mathrm{ha}^{-1}$ & + & 6 & 7.2 & $86,950 \pm 3,994$ & $4 \mathrm{a}$ & 7.0 & $112,968 \pm 5,599$ & $\mathrm{a}$ & 7,8 & $109,688 \pm 7,260$ & & 7.6 & $88,945 \pm 3,893$ & $\mathrm{a}$ \\
\hline WR, $15 \mathrm{t} \mathrm{ha}^{-1}$ & + & 6 & 7.4 & $78,396 \pm 5,631$ & $1 \mathrm{a}$ & 7.2 & $101,994 \pm 4,806$ & $\mathrm{a}$ & 7.9 & $43,947 \pm 16,737$ & $7 \mathrm{bc}$ & 7.7 & $82,788 \pm 796$ & $a b$ \\
\hline $\mathrm{WR}, 30 \mathrm{t} \mathrm{ha}^{-1}$ & + & 6 & & - & & & - & & 7.8 & $72,440 \pm 25,320$ & $0 \mathrm{ab}$ & 7.7 & $119,066 \pm 13,879$ & \\
\hline $\mathrm{TM}, 2 \mathrm{gm}^{-2}$ & - & 6 & 8 & $0 \pm 0$ & $\mathrm{~b}$ & 7.7 & $0 \pm 0$ & c & 7.8 & $0 \pm 0$ & $\mathrm{~d}$ & 7.8 & $0 \pm 0$ & c \\
\hline $\begin{array}{l}\text { BCp, } 15 \mathrm{tha}^{-1}-\mathrm{No} \\
\text { anaerobic control }\end{array}$ & - & 6 & 8 & $0 \pm 0$ & $\mathrm{~b}$ & 7.5 & $0 \pm 0$ & c & 7.7 & $0 \pm 0$ & d & 8.0 & $0 \pm 0$ & $\mathrm{c}$ \\
\hline INT-Anaerobic control & + & 6 & 7.4 & $353 \pm 353$ & $\mathrm{~b}$ & 7.5 & $1916 \pm 1,057$ & $\mathrm{c}$ & 7.9 & $2,311 \pm 643$ & $\mathrm{~cd}$ & 7.9 & $2,372 \pm 754$ & c \\
\hline INT-Standard control & - & 6 & - & $0 \pm 0$ & $\mathrm{~b}$ & 7.7 & $0 \pm 0$ & $c$ & 7.8 & $0 \pm 0$ & $\mathrm{~d}$ & 7.7 & $0 \pm 0$ & c \\
\hline
\end{tabular}

a Comp: Compost; BCp: Brassica carinata pellet; WR: Diplotaxis green manure; TM: Tolclofos methyl; INT-anaerobic control: Inoculated untreated control with anaeorobic conditions; INT-Standard control: Inoculated untreated control without aneorobic conditions.

$\mathrm{b}$ - indicates not tested.

${ }^{c}$ Means accompanied by the same letter are not significantly different $(P \leq 0.05)$, Tukey test.

between 1,017 and $1,914 \mathrm{mVh}$ in the sandy-loam soil. The cumulative anaerobic conditions of the ASD basedComp were increased in the peat soil at $26^{\circ} \mathrm{C}$ and $31^{\circ} \mathrm{C}$ after 3 weeks to, respectively, 12,532 $\mathrm{mVh}$ and 13,667 $\mathrm{mVh}$, while the cumulative values in the sandy loam soil after 3 weeks from this treatment at $26^{\circ} \mathrm{C}$ and $31^{\circ} \mathrm{C}$ 
were, respectively, 2,606 and 2,805 $\mathrm{mVh}$ (Table 2). These values were greater in the sandy-loam soil after 6 weeks of ASD application with values between $15,516 \mathrm{mVh}$ at $26^{\circ} \mathrm{C}$ and $31,817 \mathrm{mVh}$ at $31^{\circ} \mathrm{C}$.

The $\mathrm{pH}$ values of the soils were similar in both trials immediately after the ASD treatment. The $\mathrm{pH}$ increased from 6.4 to 6.7-7.1 after the $\mathrm{BCp}$ and WR green manure treatments were used as the C-sources and mixed in the peat soil, and were slightly reduced from 7.8 to 7.5 7.7 after the Brassica carbon sources were applied to the sandy-loam soil, compared to the INT-Standard control (Table 2).

Effects of soil treatments on the pathogen and plant fresh weights

The soil type and crop cycle both significantly $(P<$ 0.05 ) influenced the $D I$ and the $F W$ in both the trials, and these results are presented separately for the peat soil and sandy-loam soil, as well as for Cycles I and II, in Tables $5 \mathrm{~A}$ and $5 \mathrm{~B}$ to Tables $8 \mathrm{~A}$ and $8 \mathrm{~B}$.

Carbon source, temperature and incubation period, and the interactions between temperature $\times$ ASD duration (3 or 6 weeks), carbon source $\times$ ASD duration, and carbon source $\times$ temperature generally influenced $(P<$ $0.05)$ the percentage of lettuce plants affected by $R$. solani $(D I)$ and the harvested lettuce plant fresh weights $(F W)$, in the first and second cycles in both trials (Tables 3 and 4). The only exception for the temperature $\times$ ASD duration interaction was in the second crop cycle of Trial 2 carried out in the peat soil and in the sandy-loam soil for the tested temperatures and incubation periods (Table 3). The interactions of carbon source $\times$ ASD duration and C-source $\times$ temperature affected $D I$ and $F W$ in both soils and trials at the end of the first crop cycle (Supplementary Tables S1 and S2). The three-way interaction between these factors influenced $(P<0.001) D I$ and $F W$ in the first and second cycles in both trials and soils (Table 3).

The infested non-treated pots without anaerobic conditions (INT-Standard control) showed very high incidence of lettuce basal rot. In the first cycle, $82-90 \%$ of the plants were affected in the peat soil kept for 3 or 6 weeks at $21^{\circ} \mathrm{C}$ or $26^{\circ} \mathrm{C}$, with a slight reduction in DI at the end of Trial 2 when peat soil was kept at $31^{\circ} \mathrm{C}$ for 3 or 6 weeks (49-51\% of affected plants) (Tables $5 \mathrm{~A}$ and $5 B)$. In Trial 1, the DI for plants grown in the peat soil in the first crop cycle in the INT-Anaerobic control at $21^{\circ} \mathrm{C}$ for 6 weeks was reduced $(P<0.05)$ by $28 \%$, and at $26^{\circ} \mathrm{C}$ by $42 \%$, compared to INT-Standard control. No differences were observed between the infested untreated control, under standard and anaerobic conditions in Trial 2, for either of the tested soils and temperature conditions (Table 5B). The control treatment based on TM reduced the mean DI to a range of 63 to $83 \%$ in the peat soil ( $5 \mathrm{~A}$ and $5 \mathrm{~B})$, with a consistent effect also in the second crop cycle.

ASD based-BCp provided $60-69 \%$ disease reduction efficacy when incubated for 3 weeks at $21^{\circ} \mathrm{C}$ or $26^{\circ} \mathrm{C}$, without any effect of the extension of the treatment for 6 weeks in Trial 1 at the first crop cycle. A

Table 3. Statistical significance values for the factors ASD duration $\left(0,3\right.$ or 6 weeks), temperature $\left(21,26\right.$ or $\left.31^{\circ} \mathrm{C}\right)$, or carbon sources, for lettuce basal rot incidence ( $\%$ affected plants) and lettuce fresh weights, after trial cycles I and II.

\begin{tabular}{|c|c|c|c|c|c|c|c|c|c|}
\hline \multirow{3}{*}{ Trial } & \multirow{3}{*}{ Fixed factor } & \multicolumn{4}{|c|}{ Cycle I } & \multicolumn{4}{|c|}{ Cycle II } \\
\hline & & \multicolumn{2}{|c|}{$\begin{array}{l}\text { Sign. \% affected } \\
\text { plants }\end{array}$} & \multicolumn{2}{|c|}{$\begin{array}{c}\text { Sign. } \\
\text { Fresh weight }\end{array}$} & \multicolumn{2}{|c|}{$\begin{array}{l}\text { Sign. } \% \text { affected } \\
\text { plants }\end{array}$} & \multicolumn{2}{|c|}{$\begin{array}{c}\text { Sign. } \\
\text { Fresh weight }\end{array}$} \\
\hline & & Peat & $\begin{array}{l}\text { Sandy- } \\
\text { loam }\end{array}$ & Peat & $\begin{array}{l}\text { Sandy- } \\
\text { loam }\end{array}$ & Peat & $\begin{array}{l}\text { Sandy- } \\
\text { loam }\end{array}$ & Peat & $\begin{array}{c}\text { Sandy- } \\
\text { loam }\end{array}$ \\
\hline \multicolumn{10}{|l|}{1} \\
\hline & Carbon source & $<0.001$ & $<0.001$ & $<0.001$ & $<0.001$ & $<0.001$ & $<0.001$ & $<0.001$ & $<0.001$ \\
\hline & Temperature $\times$ ASD duration & $<0.001$ & 0.006 & $<0.001$ & $<0.001$ & 0.0816 & $<0.001$ & 0.103 & $<0.001$ \\
\hline & Carbon source $\times$ ASD duration & $<0.001$ & $<0.001$ & $<0.001$ & $<0.001$ & $<0.001$ & $<0.001$ & $<0.001$ & $<0.001$ \\
\hline & Carbon source $\times$ Temperature & $<0.001$ & $<0.001$ & $<0.001$ & $<0.001$ & $<0.001$ & $<0.001$ & $<0.001$ & $<0.001$ \\
\hline & Carbon source $\times$ Temperature $\times$ ASD duration & $<0.001$ & $<0.001$ & $<0.001$ & $<0.001$ & 0.0032 & $<0.001$ & $<0.001$ & $<0.001$ \\
\hline \multirow[t]{5}{*}{2} & Carbon source & $<0.001$ & $<0.001$ & $<0.001$ & $<0.001$ & $<0.001$ & $<0,001$ & $<0.001$ & $<0,001$ \\
\hline & Temperature $\times$ ASD duration & $<0.001$ & 0.015 & $<0.001$ & $<0.001$ & $<0.001$ & 0.0163 & $<0.001$ & 0,416 \\
\hline & Carbon source $\times$ ASD duration & $<0.001$ & $<0.001$ & $<0.001$ & $<0.001$ & 0.001 & $<0.001$ & $<0.001$ & $<0.001$ \\
\hline & Carbon source $\times$ Temperature & $<0.001$ & $<0.001$ & $<0.001$ & $<0.001$ & 0.001 & $<0.001$ & $<0.001$ & $<0.001$ \\
\hline & Carbon source $\times$ Temperature $\times$ ASD duration & $<0.001$ & $<0.001$ & $<0.001$ & $<0.001$ & $<0.001$ & $<0,001$ & $<0.001$ & 0.031 \\
\hline
\end{tabular}


Table 4. Interaction between ASD duration (weeks) and temperature $\left({ }^{\circ} \mathrm{C}\right)$ in the peat or sandy-loam soils artificially infested with Rhizoctonia solani, for Trials 1 and 2. The data are expressed as A) mean \% of lettuce plants with basal rot at the end of the I and II crop cycles B), and mean fresh weights $\left(\mathrm{g} \mathrm{pot}^{-1}\right)$ at the end of the I and II crop cycles. All data represent results from trials 1 and 2.

\begin{tabular}{|c|c|c|c|c|c|c|c|c|}
\hline \multirow{3}{*}{$\begin{array}{l}\text { ASD duration } \\
\text { (weeks) } \times \\
\text { temperature } \\
\left({ }^{\circ} \mathrm{C}\right)\end{array}$} & \multicolumn{4}{|c|}{ Peat soil } & \multicolumn{4}{|c|}{ Sandy-loam soil } \\
\hline & \multicolumn{2}{|c|}{ Mean \% affected lettuce plants } & \multicolumn{2}{|c|}{$\begin{array}{l}\text { Mean plant fresh weight ( } \mathrm{g} \\
\left.\text { pot }^{-1}\right)\end{array}$} & \multicolumn{2}{|c|}{ Mean \% affected lettuce plants } & \multicolumn{2}{|c|}{$\begin{array}{l}\text { Mean plant fresh weight ( } \mathrm{g} \\
\text { pot }^{-1} \text { ) }\end{array}$} \\
\hline & Cycle I & Cycle II & Cycle I & Cycle II & Cycle I & Cycle II & Cycle I & Cycle II \\
\hline \multicolumn{9}{|l|}{ Trial 1} \\
\hline 3 weeks at $21^{\circ} \mathrm{C}$ & $54.4 \pm 8.2 \mathrm{c}^{\mathrm{a}}$ & $50.0 \pm 6.6 \mathrm{c}$ & $95.7 \pm 24.3 \mathrm{bc}$ & $60.4 \pm 11.0 \mathrm{a}$ & $38.8 \pm 5.7 \mathrm{a}$ & $24.8 \pm 3.2 \mathrm{a}$ & $49.9 \pm 7.4 \quad b$ & $190.4 \pm 9.8 \mathrm{a}$ \\
\hline 3 weeks at $26^{\circ} \mathrm{C}$ & $50.2 \pm 7.4 \quad b$ & $39.7 \pm 5.7 \mathrm{ab}$ & $67.7 \pm 14.8 \mathrm{c}$ & $68.7 \pm 10.0 \mathrm{a}$ & $39.5 \pm 6.3$ a & $37.0 \pm 3.5 \mathrm{bc}$ & $52.8 \pm 8.6 \mathrm{~b}$ & $131.7 \pm 8.9 \mathrm{c}$ \\
\hline 6 weeks at $21^{\circ} \mathrm{C}$ & $44.4 \pm 6.9 \quad b$ & $43.4 \pm 6.3$ bc & $117.5 \pm 20.0 \mathrm{ab}$ & $74.1 \pm 14.1 \mathrm{a}$ & $47.8 \pm 6.8 \quad b$ & $29.8 \pm 3.4 \mathrm{ab}$ & $44.9 \pm 8.0 \quad b$ & $161.4 \pm 7.5 \mathrm{~b}$ \\
\hline 6 weeks at $26^{\circ} \mathrm{C}$ & $33.4 \pm 5.7 \quad$ a & $33.8 \pm 5.2$ a & $140.7 \pm 25.5 \mathrm{a}$ & $58.6 \pm 6.7$ a & $43.6 \pm 7.0 \mathrm{ab}$ & $41.7 \pm 4.3 \mathrm{c}$ & $65.4 \pm 10.8 \mathrm{a}$ & $151.7 \pm 9.2 \mathrm{bc}$ \\
\hline \multicolumn{9}{|l|}{ Trial 2} \\
\hline 3 weeks at $21^{\circ} \mathrm{C}$ & $40.4 \pm 3.9 \mathrm{~b}$ & $39.4 \pm 3.7 \quad b$ & $90.4 \pm 33.9 \mathrm{~b}$ & $127.6 \pm 19.7 \mathrm{c}$ & $18.7 \pm 1.9 \mathrm{a}$ & $20.8 \pm 2.0 \quad \mathrm{a}$ & $77.1 \pm 4.3 \mathrm{a}$ & $13.4 \pm 6.2 \mathrm{a}$ \\
\hline 3 weeks at $31^{\circ} \mathrm{C}$ & $14.6 \pm 2.0 \quad \mathrm{a}$ & $18.9 \pm 2.3 \mathrm{a}$ & $74.0 \pm 24.2 \mathrm{~b}$ & $150.7 \pm 20.2 \mathrm{c}$ & $18.5 \pm 2.2 \mathrm{a}$ & $20.3 \pm 2.1 \quad \mathrm{a}$ & $68.0 \pm 7.4 \mathrm{ab}$ & $12.7 \pm 5.8 \mathrm{a}$ \\
\hline 6 weeks at $21^{\circ} \mathrm{C}$ & $34.0 \pm 4.2 \mathrm{~b}$ & $27.0 \pm 3.2 \mathrm{~b}$ & $114.6 \pm 18.0 \mathrm{a}$ & $356.5 \pm 26.5 \mathrm{a}$ & $21.1 \pm 2.3 \mathrm{ab}$ & $21.9 \pm 2.3 \mathrm{ab}$ & $60.3 \pm 6.1 \quad b$ & $10.2 \pm 4.6 \mathrm{a}$ \\
\hline 6 weeks at $31^{\circ} \mathrm{C}$ & $21.5 \pm 3.4 \mathrm{a}$ & $11.5 \pm 2.4 \mathrm{a}$ & $119.5 \pm 23.4 \mathrm{a}$ & $251.7 \pm 25.3 b$ & $22.5 \pm 2.8 \quad b$ & $24.0 \pm 2.9 \mathrm{~b}$ & $79.9 \pm 8.3 \mathrm{a}$ & $16.0 \pm 7.5 \mathrm{a}$ \\
\hline
\end{tabular}

a Means in each column accompanied by the same letter are not significantly different for Tukey test $(P \leq 0.05)$ for cycle I and cycle II in each trial. Standard errors are also indicated.

Table 5A. Mean percentages of lettuce plants affected by basal rot after application of different ASD soil treatments, carried out for 3 or 6 weeks, and at 21 or $26^{\circ} \mathrm{C}$ in peat artificially infested with Rhizoctonia solani, at the end of the first and second crop cycles (Trial 1).

\begin{tabular}{|c|c|c|c|c|c|c|c|c|c|c|}
\hline \multirow{2}{*}{ Carbon source ${ }^{\mathrm{a}}$, dosage } & \multirow{2}{*}{ ASD } & \multirow{2}{*}{$\begin{array}{c}\text { Treatment } \\
\text { duration } \\
\text { (weeks) }\end{array}$} & \multicolumn{4}{|c|}{ Cycle I } & \multicolumn{4}{|c|}{ Cycle II } \\
\hline & & & $21^{\circ} \mathrm{C}$ & $\mathrm{E} \%{ }^{\mathrm{c}}$ & $26^{\circ} \mathrm{C}$ & $\mathrm{E} \%$ & $21^{\circ} \mathrm{C}$ & $\mathrm{E} \%$ & $26^{\circ} \mathrm{C}$ & E\% \\
\hline Comp, $15 \mathrm{t} \mathrm{ha}^{-1}$ & + & 3 & $90.0 \pm 0.0 \mathrm{~d}^{\mathrm{b}}$ & 0.0 & $76.5 \pm 4.9$ ef & 6.4 & $49.3 \pm 0.8 \quad b-d^{a}$ & 45.2 & $33.9 \pm 1.5 \mathrm{bc}$ & 52.1 \\
\hline BCp, $15 \mathrm{t} \mathrm{ha}^{-1}$ & + & 3 & $28.3 \pm 1.0 \mathrm{~b}$ & 68.6 & $32.3 \pm 4.7 \mathrm{bc}$ & 60.5 & $54.8 \pm 11.8 \mathrm{~b}-\mathrm{d}$ & 39.1 & $45.0 \pm 3.1 \mathrm{c}$ & 36.4 \\
\hline WR, $15 \mathrm{tha}^{-1}$ & + & 3 & $85.4 \pm 4.6 \mathrm{~d}$ & 5.1 & $71.2 \pm 3.5 \mathrm{~d}-\mathrm{f}$ & 12.9 & $56.4 \pm 5.0 \mathrm{~b}-\mathrm{d}$ & 37.3 & $40.6 \pm 4.5 \mathrm{bc}$ & 42.7 \\
\hline $\mathrm{TM}, 2 \mathrm{~g} \mathrm{~m}^{-2}$ & - & 3 & $22.6 \pm 1.7 \mathrm{~b}$ & 74.9 & $15.7 \pm 1.6 \mathrm{ab}$ & 80.8 & $30.4 \pm 4.2 \mathrm{~b}$ & 66.2 & $29.6 \pm 5.9 \mathrm{bc}$ & 58.2 \\
\hline INT-Anaerobic control & + & 3 & $90.0 \pm 0.0 \mathrm{~d}$ & 0.0 & $53.1 \pm 2.8 \mathrm{~d}-\mathrm{e}$ & 35.0 & $72.0 \pm 6.1 \mathrm{de}$ & 20.0 & $66.1 \pm 8.4 \mathrm{de}$ & 6.6 \\
\hline INT-Standard control & - & 3 & $90.0 \pm 0.0 \mathrm{~d}$ & 0.0 & $81.7 \pm 8.3$ f & 0.0 & $90.0 \pm 0.0 \mathrm{e}$ & 0.0 & $70.8 \pm 2.3 \mathrm{e}$ & 0.0 \\
\hline NINT-Anaerobic control & + & 3 & $0.0 \pm 0.0 \mathrm{a}$ & 100.0 & $0.0 \pm 0.0$ a & 100.0 & $0.0 \pm 0.0$ a & 100.0 & $0.0 \pm 0.0$ a & 100.0 \\
\hline NINT-Standard control & - & 3 & $0.0 \pm 0.0 \mathrm{a}$ & 100.0 & $0.0 \pm 0.0 \mathrm{a}$ & 100.0 & $0.0 \pm 0.0 \mathrm{a}$ & 100.0 & $0.0 \pm 0.0 \mathrm{a}$ & 100.0 \\
\hline Comp, $15 \mathrm{t} \mathrm{ha}^{-1}$ & + & 6 & $61.8 \pm 1.7 \mathrm{c}$ & 28.8 & $27.1 \pm 3.2 \mathrm{bc}$ & 67.0 & $42.0 \pm 5.2 \mathrm{~b}$ & 43.1 & $30.7 \pm 6.7 \mathrm{bc}$ & 54.5 \\
\hline BCp, $15 \mathrm{t} \mathrm{ha}^{-1}$ & + & 6 & $30.5 \pm 3.4 \mathrm{~b}$ & 64.9 & $33.4 \pm 5.2 \mathrm{bc}$ & 59.4 & $38.0 \pm 6.4 \mathrm{bc}$ & 48.5 & $44.2 \pm 5.8 \mathrm{c}$ & 34.4 \\
\hline WR, $15 \mathrm{tha}^{-1}$ & + & 6 & $56.0 \pm 7.9$ с & 35.5 & $46.6 \pm 4.6 \mathrm{~cd}$ & 43.3 & $42.7 \pm 4.6 \mathrm{bc}$ & 42.1 & $37.7 \pm 1.9 \mathrm{bc}$ & 44.1 \\
\hline $\mathrm{TM}, 2 \mathrm{~g} \mathrm{~m}^{-2}$ & - & 6 & $15.6 \pm 5.9 \mathrm{ab}$ & 82.0 & $19.5 \pm 1.1 \mathrm{ab}$ & 76.3 & $31.5 \pm 2.1 \mathrm{~b}$ & 57.3 & $23.6 \pm 1.9 \mathrm{~b}$ & 65.0 \\
\hline INT-Anaerobic control & + & 6 & $62.2 \pm 6.3 \mathrm{c}$ & 28.3 & $47.9 \pm 2.0 \mathrm{~cd}$ & 41.7 & $71.3 \pm 6.7 \mathrm{de}$ & 3.4 & $48.0 \pm 4.3 \mathrm{~cd}$ & 28.8 \\
\hline INT-Standard control & - & 6 & $86.8 \pm 3.2 \mathrm{~d}$ & 0.0 & $82.2 \pm 4.7 \mathrm{f}$ & 0.0 & $73.8 \pm 6.4 \mathrm{de}$ & 0.0 & $67.4 \pm 1.7 \mathrm{de}$ & 0.0 \\
\hline NINT-Anaerobic control & + & 6 & $0.0 \pm 0.0 \mathrm{a}$ & 100.0 & $0.0 \pm 0.0 \mathrm{a}$ & 100.0 & $0.0 \pm 0.0 \mathrm{a}$ & 100.0 & $0.0 \pm 0.0$ a & 100.0 \\
\hline NINT-Standard control & - & 6 & $0.0 \pm 0.0 \mathrm{a}$ & 100.0 & $0.0 \pm 0.0 \mathrm{a}$ & 100.0 & $0.0 \pm 0.0 \mathrm{a}$ & 100.0 & $0.0 \pm 0.0 \mathrm{a}$ & 100.0 \\
\hline
\end{tabular}

a Comp: Compost; BCp: Brassica carinata pellet with anaerobic conditions; WR: Diplotaxis green manure; TM: Tolclofos methyl; INTAnaerobic control: Inoculated untreated control with anaerobic conditions; INT-Standard control: Inoculated untreated control without anaerobic conditions; NINT-Anaerobic control: Non-inoculated untreated control with anaerobic conditions; NINT-Standard control: Noninoculated untreated control without anaerobic conditions.

${ }^{\mathrm{b}}$ Means in each column accompanied by the same letter are not significantly different $(P \leq 0.05)$, according to Tukey's test. Standard errors are also indicated.

${ }^{c} \mathrm{E} \%$ : Disease reduction compared to the INT-Standard controls carried out for 3 or 6 weeks. 
Table 5B. Mean percentages of lettuce plants affected by basal rot after application of different ASD soil treatments, carried out for 3 or 6 weeks, and at 21 or $31^{\circ} \mathrm{C}$ in peat artificially infested with Rhizoctonia solani, at the end of the first and second crop cycles (Trial 2).

\begin{tabular}{|c|c|c|c|c|c|c|c|c|c|c|c|c|c|}
\hline \multirow{2}{*}{ Carbon source $^{\mathrm{a}}$, dosage } & \multirow{2}{*}{ ASD } & \multirow{2}{*}{$\begin{array}{c}\text { Treatment } \\
\text { duration } \\
\text { (weeks) }\end{array}$} & \multicolumn{6}{|c|}{ Cycle I } & \multicolumn{5}{|c|}{ Cycle II } \\
\hline & & & $21^{\circ} \mathrm{C}$ & & $\mathrm{E} \%{ }^{\mathrm{c}}$ & $26^{\circ} \mathrm{C}$ & & $\mathrm{E} \%$ & $21^{\circ} \mathrm{C}$ & & $\mathrm{E} \%$ & $26^{\circ} \mathrm{C}$ & $\mathrm{E} \%$ \\
\hline Comp, $15 \mathrm{t} \mathrm{ha}^{-1}$ & + & 3 & $86.8 \pm 3.2$ & $\mathrm{~cd}^{\mathrm{b}}$ & 3.6 & $39.0 \pm 4.4$ & $c-e$ & 21.1 & $38.5 \pm 0.7$ & $c-g$ & 27.5 & $67.0 \pm 8.4 \quad b-e$ & 25.6 \\
\hline BCp, $15 \mathrm{t} \mathrm{ha}^{-1}$ & + & 3 & $45.3 \pm 2.0$ & $\mathrm{ab}$ & 49.7 & $23.71 \pm 1.1$ & b-e & 52.0 & $36.14 \pm 7.9$ & $c-g$ & 32.0 & $63.1 \pm 15.5 \mathrm{~b}-\mathrm{e}$ & 29.9 \\
\hline WR, $15 \mathrm{tha}^{-1}$ & + & 3 & $80.1 \pm 6.3$ & $b-d$ & 11.0 & $38.2 \pm 5.1$ & $c-e$ & 22.7 & $40.6 \pm 4.5$ & d-g & 23.5 & $42.8 \pm 4.2 \quad \mathrm{a}-\mathrm{c}$ & 52.4 \\
\hline $\mathrm{WR}, 30 \mathrm{t} \mathrm{ha}^{-1}$ & + & 3 & $84.3 \pm 5.7$ & $b-d$ & 6.3 & $30.11 \pm 0.5$ & b-e & 39.1 & $43.6 \pm 3.8$ & d-g & 17.9 & $59.2 \pm 15.2 \mathrm{a}-\mathrm{d}$ & 34.2 \\
\hline $\mathrm{TM}, 2 \mathrm{gm}^{-2}$ & - & 3 & $19.7 \pm 4.2$ & $\mathrm{a}$ & 78.1 & $14.6 \pm 5.4$ & $a-c$ & 70.4 & $13.4 \pm 21.0$ & $a-c$ & 74.8 & $0.0 \pm 0.0 \quad \mathrm{a}$ & 100.0 \\
\hline $\begin{array}{l}\mathrm{BCp}, 15 \mathrm{t} \mathrm{ha}^{-1}-\mathrm{No} \\
\text { anaerobic control }\end{array}$ & - & 3 & $90.0 \pm 0.0$ & $\mathrm{~d}$ & 0.0 & $38.8 \pm 6.1$ & $c-e$ & 21.5 & $42.8 \pm 2.2$ & d-g & 19.4 & $67.5 \pm 13.9$ b-e & 25.0 \\
\hline INT- Anaerobic control & + & 3 & $90.0 \pm 0.0$ & d & 0.0 & $47.9 \pm 1.7$ & de & 3.0 & $54.7 \pm 3.6$ & g & 0.0 & $82.5 \pm 7.5 \mathrm{de}$ & 8.3 \\
\hline INT- Standard control & - & 3 & $90.0 \pm 0.0$ & $\mathrm{~d}$ & 0.0 & $49.4 \pm 1.9$ & de & 0.0 & $53.1 \pm 2.6$ & fg & 0.0 & $90.0 \pm 0.0 \mathrm{e}$ & 0.0 \\
\hline NINT- Anaerobic control & $1+$ & 3 & $0.0 \pm 0.0$ & $\mathrm{a}$ & 100.0 & $0.0 \pm 0.0$ & $\mathrm{a}$ & 100.0 & $0.0 \pm 0.0$ & $\mathrm{a}$ & 100.0 & $0.0 \pm 0.0$ & 100.0 \\
\hline Comp, $15 \mathrm{t} \mathrm{ha}^{-1}$ & + & 6 & $57.3 \pm \pm 4.7$ & $\mathrm{~b}$ & 28.1 & $40.6 \pm 2.8$ & $c-e$ & 20.1 & $28.2 \pm 1.8$ & $c-e$ & 31.6 & $31.6 \pm 0.9$ & 48.2 \\
\hline BCp, $15 \mathrm{t} \mathrm{ha}^{-1}$ & + & 6 & $53.8 \pm 2.9$ & $\mathrm{~b}$ & 32.5 & $10.7 \pm 7.1$ & $\mathrm{ab}$ & 78.9 & $3.2 \pm 3.2$ & $\mathrm{ab}$ & 92.2 & $33.9 \pm 1.5$ & 44.4 \\
\hline WR, $15 \mathrm{tha}^{-1}$ & + & 6 & $71.4 \pm 6.9$ & $b-d$ & 10.4 & $8.3 \pm 8.3$ & $\mathrm{a}$ & 83.7 & $21.3 \pm 2.9$ & $a-e$ & 48.3 & $28.9 \pm 5.4$ & 52.6 \\
\hline $\mathrm{WR}, 30 \mathrm{tha}^{-1}$ & + & 6 & $64.1 \pm 10.5$ & $\mathrm{bc}$ & 19.6 & $11.4 \pm 6.6$ & $\mathrm{ab}$ & 77.6 & $16.8 \pm 2.4$ & $a-c$ & 59.2 & $30.3 \pm 8.6$ & 50.3 \\
\hline $\mathrm{TM}, 2 \mathrm{gm}^{-2}$ & - & 6 & $21.3 \pm 2.2$ & $\mathrm{a}$ & 73.3 & $17.9 \pm 6.3$ & a-e & 64.8 & $8.6 \pm 3.0$ & $\mathrm{ab}$ & 79.1 & $24.4 \pm 5.2 \mathrm{ab}$ & 60.0 \\
\hline $\begin{array}{l}\text { BCp, } 15 \mathrm{t} \mathrm{ha}^{-1} \text { - No } \\
\text { anaerobic control }\end{array}$ & - & 6 & $69.3 \pm 14.2$ & $b-d$ & 13.0 & $36.1 \pm 2.8$ & $c-e$ & 28.9 & $29.5 \pm 3.9$ & $c-e$ & 28.4 & $68.9 \pm 10.6 \mathrm{c}-\mathrm{e}$ & -13.0 \\
\hline INT- Anaerobic control & + & 6 & 70.58 .9 & $b-d$ & 11.5 & $39.3 \pm 2.4$ & de & 22.6 & $32.1 \pm 4.4$ & $c-f$ & 22.1 & $63.1 \pm 13.3$ b-e & -3.4 \\
\hline INT- Standard control & - & 6 & 79.76 .0 & $b-d$ & 0.0 & $50.8 \pm 1.7$ & e & 0.0 & $41.2 \pm 2.4$ & d-g & 0.0 & $61.0 \pm 2.4 \quad b-e$ & 0.0 \\
\hline NINT- Anaerobic control & $1+$ & 6 & 0.00 .0 & a & 100.0 & $0.0 \pm 0.0$ & a & 100.0 & $0.0 \pm 0.0$ & a & 100.0 & $0.0 \pm 0.0 \quad \mathrm{a}$ & 100.0 \\
\hline
\end{tabular}

a Comp: Compost; BCp: Brassica carinata pellet with or without anaerobic conditions; WR: Diplotaxis green manure; TM: Tolclofos methyl; INT-Anaerobic control: Inoculated untreated control with anaerobiotic conditions; INT-Standard control: Inoculated untreated control without anaerobic conditions; NINT-Anaerobic control: Non inoculated untreated control with anaerobic conditions.

${ }^{\mathrm{b}}$ Means in each column accompanied by a common letter are not significantly different $(P \leq 0.05)$, according to Tukey's test. Standard errors are also reported.

${ }^{c} \mathrm{E} \%$ : Disease reduction compared to the INT-Standard controls carried out for 3 or 6 weeks.

slight reduction in ASD based-BCp efficacy occurred at the end of the second cycle carried out in the same soil (34-39\% efficacy) (Table 5A). The same trend was found in the Trial 2 when ASD based-BCp was applied for 3 weeks at $21^{\circ} \mathrm{C}$ or $31^{\circ} \mathrm{C}(50-51 \%$ efficacy), with an increase in efficacy to $79 \%$ when incubated at $31^{\circ} \mathrm{C}$ for 6 weeks at the end of the first cycle. A positive effect of 6 weeks of ASD based-BCp also occurred in the second crop cycle (Table 5B). BCp applied at $15 \mathrm{t} \mathrm{ha}^{-1}$ without anaerobic conditions and tested in Trial 2, provided no statistically significant advantages when applied to the infested peat soil for any of the tested conditions (Tables 5B).

WR used as green manure and mixed with the peat soil was not effective against basal rot when applied for 3 weeks at any of the tested temperatures in Trials 1 and 2 at the end of the first cycle (Tables 5A and 5B) at both the tested dosages. This treatment applied for 6 weeks at $26^{\circ} \mathrm{C}$ reduced $(P<0.05)$ the disease by $43 \%$, and at $31^{\circ} \mathrm{C}$ by $78-84 \%$, compared to the INT-Standard control, without any effect of the dosage used.

The greatest efficacy of ASD based-Comp was observed in Trial 1 at $26^{\circ} \mathrm{C}$ for 6 weeks of incubation (67\% efficacy), while the same carbon source did not provide any effect compared to the INT-Standard control in all the tested conditions at the first crop cycle in both trials (Tables 5A and 5B). ASD based-Comp gave increased efficacy against basal rot in the second crop cycle (32 to $54 \%$ efficacy), without any significant effects of temperature or duration of the treatment (Tables $5 \mathrm{~A}$ and $5 \mathrm{~B})$.

At the end of the first cycle, the plants grown in the treated peat had generally reduced $F W$ at $21^{\circ} \mathrm{C}$ and $26^{\circ} \mathrm{C}$, and only the ASD based-Comp treatment applied for 6 weeks at $26^{\circ} \mathrm{C}$ improved $(P<0.05)$ the mean plant fresh weights, compared to the inoculated and untreated standard control (Table 6A). The lettuce plants grown in the same peat soil, previously treated with Comp and WR as C-sources for 3 or 6 weeks during the second crop cycle, at both temperatures, generally had greater 
Table 6A. Mean fresh weight $\left(\mathrm{g} \mathrm{pot}^{-1}\right)$ of lettuce plants after application of different ASD soil treatments, carried out for 3 or 6 weeks, and at 21 or $26^{\circ} \mathrm{C}$ in peat artificially infested with Rhizoctonia solani, at the end of the first and second crop cycles (Trial 1).

\begin{tabular}{|c|c|c|c|c|c|c|c|c|c|c|}
\hline \multirow{3}{*}{$\begin{array}{l}\text { Carbon source }^{\mathrm{a}} \text {, dosage } \\
\text { Comp, } 15 \mathrm{t} \mathrm{ha}^{-1}\end{array}$} & \multirow{3}{*}{$\begin{array}{c}\text { ASD } \\
+\end{array}$} & \multirow{3}{*}{$\begin{array}{c}\begin{array}{c}\text { Treatment } \\
\text { duration } \\
\text { (weeks) }\end{array} \\
3\end{array}$} & \multicolumn{4}{|c|}{ Cycle I } & \multicolumn{4}{|c|}{ Cycle II } \\
\hline & & & \multicolumn{2}{|l|}{$21^{\circ} \mathrm{C}$} & \multicolumn{2}{|l|}{$26^{\circ} \mathrm{C}$} & \multicolumn{2}{|l|}{$21^{\circ} \mathrm{C}$} & \multicolumn{2}{|l|}{$26^{\circ} \mathrm{C}$} \\
\hline & & & $2.0 \pm 0.0$ & $\mathrm{e}^{\mathrm{b}}$ & $18.0 \pm 7.6$ & c & $197.3 \pm 5.3$ & $\mathrm{a}$ & $166.1 \pm 25.2$ & a \\
\hline BCp, $15 \mathrm{t} \mathrm{ha}^{-1}$ & + & 3 & $70.0 \pm 3.7$ & $\mathrm{~cd}$ & $93.3 \pm 12.4$ & $\mathrm{bc}$ & $76.4 \pm 19.6$ & $b-d$ & $94.6 \pm 15.1$ & $b-d$ \\
\hline $\mathrm{WR}, 15 \mathrm{t} \mathrm{ha}^{-1}$ & + & 3 & $2.7 \pm 2.0$ & $\mathrm{e}$ & $9.6 \pm 3.9$ & c & $78.9 \pm 14.3$ & $b-d$ & $43.4 \pm 4.6$ & ef \\
\hline $\mathrm{TM}, 2 \mathrm{gm}^{-2}$ & - & 3 & $67.4 \pm 9.2$ & $\mathrm{~cd}$ & $84.1 \pm 2.5$ & c & $40.5 \pm 0.8$ & $\mathrm{~cd}$ & $35.3 \pm 2.0$ & $\mathrm{f}$ \\
\hline INT- Anaerobic control & + & 3 & $2.2 \pm 0.0$ & $\mathrm{e}$ & $16.4 \pm 4.0$ & c & $16.3 \pm 5.5$ & $\mathrm{~cd}$ & $29.3 \pm 13.0$ & $\mathrm{f}$ \\
\hline INT- Standard control & - & 3 & $2.6 \pm 0.0$ & e & $2.4 \pm 0.0$ & c & $3.0 \pm 2.0$ & $\mathrm{~d}$ & $17.0 \pm 4.5$ & $\mathrm{f}$ \\
\hline NINT- Anaerobic control & + & 3 & $387.6 \pm 25.6$ & $\mathrm{a}$ & $232.8 \pm 27.3$ & $\mathrm{ab}$ & $14.0 \pm 1.6$ & $\mathrm{~cd}$ & $33.0 \pm 5.1$ & $\mathrm{f}$ \\
\hline NINT- Standard control & - & 3 & $238.1 \pm 19.3$ & $\mathrm{~b}$ & $153.3 \pm 24.3$ & $\mathrm{ab}$ & $80.1 \pm 8.2$ & $b-d$ & $131.3 \pm 8.2$ & $\mathrm{ab}$ \\
\hline Comp, $15 \mathrm{t} \mathrm{ha}^{-1}$ & + & 6 & $93.21 \pm 1.8$ & c & $356.2 \pm 111.4$ & $\mathrm{a}$ & $201.7 \pm 14.1$ & $\mathrm{a}$ & $108.9 \pm 6.1$ & $\mathrm{~b}$ \\
\hline BCp, $15 \mathrm{t} \mathrm{ha}^{-1}$ & + & 6 & $52.21 \pm 1.4$ & $c-e$ & $143.6 \pm 17.8$ & bc & $163.2 \pm 59.7$ & $\mathrm{ab}$ & $35.9 \pm 2.6$ & $\mathrm{f}$ \\
\hline WR, $15 \mathrm{t} \mathrm{ha}^{-1}$ & + & 6 & $96.22 \pm 3.9$ & $\mathrm{~cd}$ & $87.8 \pm 14.1$ & $\mathrm{bc}$ & $93.7 \pm 13.6$ & $b-d$ & $105.4 \pm 8.6$ & $\mathrm{~b}$ \\
\hline $\mathrm{TM}, 2 \mathrm{gm}^{-2}$ & - & 6 & $98.7 \pm 24.7$ & $\mathrm{~cd}$ & $87.4 \pm 14.4$ & $\mathrm{bc}$ & $42.3 \pm 3.7$ & $\mathrm{~cd}$ & $58.1 \pm 11.7$ & $c-f$ \\
\hline INT- Anaerobic control & + & 6 & $27.1 \pm 8.4$ & $\mathrm{de}$ & $42.4 \pm 14.2$ & c & $16.7 \pm 6.4$ & $\mathrm{~cd}$ & $46.5 \pm 6.8$ & $d-f$ \\
\hline INT- Standard control & - & 6 & $1.8 \pm 1.0$ & $\mathrm{e}$ & $8.1 \pm 5.3$ & c & $17.0 \pm 11.6$ & $\mathrm{~cd}$ & $11.7 \pm 1.3$ & $\mathrm{f}$ \\
\hline NINT- Anaerobic control & + & 6 & $210.8 \pm 7.4$ & $\mathrm{~b}$ & $254.3 \pm 48.4$ & $\mathrm{ab}$ & $13.9 \pm 2.7$ & $\mathrm{~cd}$ & $15.8 \pm 2.0$ & $\mathrm{f}$ \\
\hline NINT- Standard control & - & 6 & 360.419 .7 & $\mathrm{a}$ & $246.1 \pm 31.5$ & $\mathrm{ab}$ & $44.3 \pm 4.4$ & $\mathrm{~cd}$ & $86.3 \pm 2.4$ & b-e \\
\hline
\end{tabular}

a Comp: Compost; BCp: Brassica carinata pellet with anaerobic conditions; WR: Diplotaxis green manure; TM: Tolclofos methyl; INTAnaerobic control: Inoculated untreated control with anaerobic conditions; INT-Standard control: Inoculated untreated control without anaerobic conditions; NINT-Anaerobic control: Non-inoculated untreated control with anaerobic conditions; NINT-Standard control: Noninoculated untreated control without anaerobic conditions.

${ }^{\mathrm{b}}$ Means in each column accompanied by the same letter are not significantly different $(P \leq 0.05)$, according to Tukey's test. Standard errors are also indicated.

fresh weights than during cycle I. The greatest mean fresh weights reached in the second crop cycle were recorded from the $\mathrm{BCp}$ and $\mathrm{WR}$ (at $15 \mathrm{t} \mathrm{ha}^{-1}$ ) carbon sources at $31^{\circ} \mathrm{C}$ for 6 weeks incubation (Table $6 \mathrm{~B}$ ).

The infested non-treated pots without anaerobiosis (INT-Standard control) had $69 \%$ to $79 \%$ of basal rot affected plants in the sandy-loam soil at the end of the first crop cycle in Trial 1 (Table 7A), and there was a slight reduction in disease incidence at the end of Trail 2 (32-44\% of affected plants) (Table 7B). The control treatment based on TM reduced the incidence of affected plants to a range of 74 to $90 \%$ in the sandy-loam soil (Tables 7A and 7B). BCp applied under anaerobic conditions in the sandy-loam soil at $21^{\circ} \mathrm{C}$, provided reduced disease (52\% in Trial 1 and $72 \%$ in Trial 2) when carried out for 3 weeks, compared to INT-Standard control. The same carbon source, applied under anaerobic conditions generally gave more consistent disease reduction when applied at $26^{\circ} \mathrm{C}$ for both 3 and 6 weeks $(52-63 \%$ efficacy), and complete disease control was provided by this treatment when it was applied for 6 weeks at $31^{\circ} \mathrm{C}$ (Table 7B). The efficacy of the ASD based-BCp was generally improved in the second crop cycle (Tables 7A and
7B). The BCp treatment applied without any anaerobic conditions in the sandy-loam soil and tested in Trial 2, reduced basal rot incidence, compared to the INT-Standard control, by $71 \%$ at $31^{\circ} \mathrm{C}$ for 6 weeks and by $44 \%$ at $21^{\circ} \mathrm{C}$ for 3 weeks (Table 7B). The ASD treatment with WR as green manure applied to the sandy-loam soil at $21^{\circ} \mathrm{C}$ for 3 weeks generally partially reduced the percentage of affected plants (32-37\% efficacy) and resulted in a greater efficacy (from 46 to $59 \%$ ) at $31^{\circ} \mathrm{C}$ (Table 7B), with no significant difference between the two dosages in Trial 2. The efficacy of the ASD treatment with WR generally increased in the second crop cycle for all the tested conditions (Tables 7A and 7B). Inconsistent results were observed when the compost was mixed into the sandyloam soil, with the greatest disease reduction $(38-41 \%$ efficacy) at $21^{\circ} \mathrm{C}$ for 3 weeks of incubation (Tables 7A and 7B). Efficacy of the ASD treatment with compost was generally increased in the second crop cycle, compared to the first, with the greatest disease reduction in the sandyloam soil previously treated for 3 weeks at $21^{\circ} \mathrm{C}(53 \%$ efficacy in Trial 1 and 66\% efficacy in Trial 2).

Mean fresh weights of the lettuce plants grown in the ASD treated sandy-loam soil at the end of the first crop 
Table 6B. Mean fresh weight $\left(\mathrm{g} \mathrm{pot}^{-1}\right)$ of lettuce plants after application of different ASD soil treatments, carried out for 3 or 6 weeks, and at 21 or $31^{\circ} \mathrm{C}$ in peat artificially infested with Rhizoctonia solani, at the end of the first and second crop cycles (Trial 2).

\begin{tabular}{|c|c|c|c|c|c|c|c|c|c|c|}
\hline \multirow{3}{*}{$\begin{array}{l}\text { Carbon source }^{\mathrm{a}} \text {, dosage } \\
\text { Comp, } 15 \mathrm{t} \mathrm{ha}^{-1}\end{array}$} & \multirow{3}{*}{$\begin{array}{c}\text { ASD } \\
+\end{array}$} & \multirow{3}{*}{$\begin{array}{c}\text { Treatment } \\
\begin{array}{c}\text { Duration } \\
\text { (Weeks) }\end{array} \\
3\end{array}$} & \multicolumn{4}{|c|}{ Cycle I } & \multicolumn{4}{|c|}{ Cycle II } \\
\hline & & & \multicolumn{2}{|l|}{$21^{\circ} \mathrm{C}$} & \multicolumn{2}{|l|}{$31^{\circ} \mathrm{C}$} & \multicolumn{2}{|l|}{$21^{\circ} \mathrm{C}$} & \multicolumn{2}{|l|}{$31^{\circ} \mathrm{C}$} \\
\hline & & & $10.5 \pm 0.5$ & $\mathrm{~b}^{\mathrm{b}}$ & $111.9 \pm 11.6$ & de & $63.4 \pm 14.5$ & $c-f$ & $208.0 \pm 4.6$ & $c-g$ \\
\hline BCp, $15 \mathrm{t} \mathrm{ha}^{-1}$ & + & 3 & $36.5 \pm 0.0$ & $\mathrm{~b}$ & $246.5 \pm 81.7$ & $a-d$ & $32.7 \pm 10.1$ & $d-f$ & $162.0 \pm 71.0$ & d-g \\
\hline WR, $15 \mathrm{t} \mathrm{ha}^{-1}$ & + & 3 & $13.0 \pm 12.6$ & $\mathrm{~b}$ & $287.4 \pm 74.8$ & $a-d$ & $41.3 \pm 13.1$ & $d-f$ & $198.0 \pm 8.3$ & $c-g$ \\
\hline $\mathrm{WR}, 30 \mathrm{t} \mathrm{ha}^{-1}$ & + & 3 & $11.0 \pm 1.0$ & $\mathrm{~b}$ & $79.6 \pm 6.1$ & $\mathrm{e}$ & $141.6 \pm 7.0$ & $a-d$ & $281.5 \pm 10.4$ & $b-f$ \\
\hline $\mathrm{TM}, 2 \mathrm{gm}^{-2}$ & - & 3 & $102.2 \pm 28.1$ & $\mathrm{a}$ & $373.65 \pm 0.1$ & $a-c$ & $192.4 \pm 15.0$ & $\mathrm{ab}$ & $305.4 \pm 107.4$ & $b-e$ \\
\hline BCp, 15 t ha-1- No anaerobic control & - & 3 & $10.3 \pm 0.3$ & $\mathrm{~b}$ & $97.4 \pm 31.1$ & de & $97.9 \pm 9.2$ & $b-f$ & $47.5 \pm 4.8$ & fg \\
\hline INT- Anaerobic control & + & 3 & $10.0 \pm 0.0$ & $\mathrm{~b}$ & $69.6 \pm 23.7$ & $\mathrm{e}$ & $13.8 \pm 1.9$ & $\mathrm{f}$ & $42.7 \pm 24.7$ & $\mathrm{~g}$ \\
\hline INT- Standard control & - & 3 & $12.3 \pm 12.3$ & $\mathrm{~b}$ & $73.2 \pm 13.1$ & $\mathrm{e}$ & $4.0 \pm 0.0$ & $\mathrm{f}$ & $47.4 \pm 25.0$ & fg \\
\hline NINT- Anaerobic control & + & 3 & $117.4 \pm 24.0$ & a & $233.7 \pm 13.9$ & b-e & $206.21 \pm 2.1$ & a & $301 \pm 31.8$ & b-e \\
\hline Comp, $15 \mathrm{t} \mathrm{ha}^{-1}$ & + & 6 & $28.4 \pm 10.6$ & $\mathrm{~b}$ & $99.9 \pm 14.5$ & de & $132.0 \pm 3.8$ & a-e & $172.5 \pm 25.0$ & $c-g$ \\
\hline BCp, $15 \mathrm{t} \mathrm{ha}^{-1}$ & + & 6 & $13.1 \pm 1.8$ & $\mathrm{~b}$ & $419.0 \pm 39.5$ & $\mathrm{ab}$ & $195.2 \pm 7.5$ & $\mathrm{ab}$ & $657.1 \pm 45.4$ & \\
\hline WR, $15 \mathrm{t} \mathrm{ha}^{-1}$ & + & 6 & $15.6 \pm 6.5$ & $\mathrm{~b}$ & $460.3 \pm 31.8$ & $\mathrm{ab}$ & $124.81 \pm 1.9$ & b-e & $405.4 \pm 98.7$ & bc \\
\hline $\mathrm{WR}, 30 \mathrm{t} \mathrm{ha}^{-1}$ & + & 6 & $33.9 \pm 17.5$ & $\mathrm{~b}$ & $186.5 \pm 50.6$ & c-e & $165.5 \pm 25.0$ & $a-c$ & $497.4 \pm 52.6$ & $\mathrm{ab}$ \\
\hline $\mathrm{TM}, 2 \mathrm{gm}^{-2}$ & - & 6 & $96.4 \pm 20.0$ & $\mathrm{a}$ & $376 \pm 55.6$ & $a-c$ & $99.1 \pm 9.1$ & $b-f$ & $334.5 \pm 31.7$ & $b-d$ \\
\hline BCp, $15 \mathrm{t} \mathrm{ha}^{-1}$ - No anaerobic control & - & 6 & $16.9 \pm 4.0$ & $\mathrm{~b}$ & $113.5 \pm 5.4$ & de & $12.5 \pm 3.4$ & f & $294.2 \pm 42.6$ & $b-e$ \\
\hline INT- Anaerobic control & + & 6 & $15.7 \pm 9.5$ & $\mathrm{~b}$ & $101.3 \pm 7.8$ & de & $25.0 \pm 8.7$ & ef & $73.8 \pm 8.6$ & e-g \\
\hline INT- Standard control & - & 6 & $29.2 \pm 4.0$ & $\mathrm{~b}$ & $70.51 \pm 7.7$ & $\mathrm{e}$ & $23.0 \pm 3.0$ & ef & $89.5 \pm 18.9$ & e-g \\
\hline NINT- Anaerobic control & + & 6 & $152.9 \pm 14.1$ & $\mathrm{a}$ & $221 \pm 13.5$ & $c-e$ & $132.7 \pm 10.1$ & b-e & $239.3 \pm 20.4$ & $c-g$ \\
\hline
\end{tabular}

a Comp: Compost; BCp: Brassica carinata pellet with or without anaerobic conditions; WR: Diplotaxis green manure; TM: Tolclofos methyl; INT-Anaerobic control: Inoculated untreated control with anaerobiotic conditions; INT-Standard control: Inoculated untreated control without anaerobic conditions; NINT-Anaerobic control: Non inoculated untreated control with anaerobic conditions.

${ }^{\mathrm{b}}$ Means in each column accompanied by a common letter are not significantly different $(P \leq 0.05)$, according to Tukey's test. Standard errors are also reported.

cycle were very low for all the treatments (Tables $8 \mathrm{~A}$ and $8 \mathrm{~B}$ ). Moreover, there was a significant reduction in the mean lettuce fresh weights in the non-infested control (NINT-Anaerobic control) incubated for 6 weeks. By the end of the second crop cycle, lettuce plants generally had significantly greater fresh weights than observed after the first cycle. The greatest mean fresh weight was at $21^{\circ} \mathrm{C}$ using WR at $15 \mathrm{t} \mathrm{ha}^{-1}$ for 3 weeks and BCp for 6 weeks (Table 8A). The lettuce plants of the second crop cycle grown in the same sandy-loam soil, previously treated at $31^{\circ} \mathrm{C}$ for 6 weeks using WR at both the tested dosages and BCp, generally had significantly greater mean fresh weights than the non-inoculated control plants under anaerobic conditions (Table 8B).

\section{DISCUSSION}

ASD has been proposed in several reports as a possible solution for soil disinfestation to control several plant pathogens, on different crops, using a variety of $\mathrm{C}$-sources, under controlled conditions and in field experiments (Momma et al., 2006; Katase et al., 2009; Butler et al., 2012 a, b; Runia et al., 2012; 2014; Rosskopf et al., 2015; Strauss and Kluepfel, 2015; Hewavitharana and Mazzola, 2016; Shrestha et al., 2016; Shennan et al., 2014; 2018). Since this methodology requires further testing before practical implementation, considerable efforts have aimed to improve ASD efficacy with emphasis on the optimizing factors such as carbon sources (Butler et al., 2012a; b; 2014b; Hewavitharana et al., 2014; Shrestha et al., 2016; Rodríguez-Molina et al., 2016; Serrano-Pérez et al., 2017), duration of the incubation periods, and soil temperatures (Hewavitharana et al., 2015; Shrestha et al., 2016; Shennan et al., 2014; 2018) for developing standard treatments against specific soilborne pathogens. For example, Runia et al., (2012; 2014) set up a controlled laboratory system to simulate ASD treatments in mesocosms to study biotic and abiotic changes over time against Verticillium dahliae and Globodera pallida. A similar approach has also been used for other pathosystems, including Phytophthora nicotianae-pepper (Serrano-Pérez et al., 2017) and Verticillium dahliae-strawberry (Shennan et al., 2018). 
Table 7A. Mean percentages of lettuce plants affected by basal rot after application of different ASD soil treatments, carried out for 3 or 6 weeks, and at 21 or $26{ }^{\circ} \mathrm{C}$ in sandy-loam artificially infested with Rhizoctonia solani, at the end of the first and second crop cycles (Trial 1).

\begin{tabular}{|c|c|c|c|c|c|c|c|c|c|c|c|c|}
\hline \multirow{2}{*}{ Carbon source ${ }^{\mathrm{a}}$, dosage } & \multirow{2}{*}{ ASD } & \multirow{2}{*}{$\begin{array}{l}\text { Treatment } \\
\text { Duration } \\
\text { (Weeks) }\end{array}$} & \multicolumn{4}{|c|}{ Cycle I } & \multicolumn{6}{|c|}{ Cycle II } \\
\hline & & & $21^{\circ} \mathrm{C}$ & $\mathrm{E} \%{ }^{\mathrm{c}}$ & $26^{\circ} \mathrm{C}$ & $\mathrm{E} \%$ & $21^{\circ} \mathrm{C}$ & & E\% & $26^{\circ} \mathrm{C}$ & & $\mathrm{E} \%$ \\
\hline Comp, $15 \mathrm{t} \mathrm{ha}^{-1}$ & + & 3 & $45.0 \pm 1.2 \mathrm{~cd}^{\mathrm{b}}$ & 41.2 & $47.3 \pm 4.4 \mathrm{de}$ & 34.5 & $19.9 \pm 3.6$ & $\mathrm{bc}$ & 66.0 & $35.36 \pm 3.3$ & $b-d$ & 27.8 \\
\hline BCp, $15 \mathrm{t} \mathrm{ha}^{-1}$ & + & 3 & $37.0 \pm 1.9 \mathrm{c}$ & 51.6 & $27.1 \pm 3.2 \mathrm{~b}-\mathrm{d}$ & 62.5 & $33.2 \pm 1.3$ & $c d$ & 43.3 & $42.86 \pm 3.6$ & $c-e$ & 55.5 \\
\hline WR, $15 \mathrm{t} \mathrm{ha}^{-1}$ & + & 3 & $48.6 \pm 2.2$ c-e & 36.5 & $58.3 \pm 6.4$ ef & 19.3 & $27.8 \pm 3.7$ & $\mathrm{bc}$ & 52.6 & $52.74 \pm 5.2$ & de & 46.3 \\
\hline $\mathrm{TM}, 2 \mathrm{gm}^{-2}$ & - & 3 & $15.7 \pm 1.6 \mathrm{~b}$ & 79.5 & $16.8 \pm 2.4 \mathrm{a}-\mathrm{c}$ & 76.7 & $16.8 \pm 2.4$ & $\mathrm{~b}$ & 71.3 & $20.41 \pm 7.5$ & $a-c$ & 74.0 \\
\hline INT- Anaerobic control & + & 3 & $67.4 \pm 1.7 \mathrm{fg}$ & 11.9 & $59.4 \pm 2.9$ ef & 17.7 & $46.5 \pm 3.9$ & $d-f$ & 20.6 & $56.18 \pm 2.7$ & de & 19.9 \\
\hline INT- Standard control & - & 3 & $76.5 \pm 4.9 \mathrm{~g}$ & 0.0 & $72.2 \pm 6.4 \mathrm{f}$ & 0.0 & $58.6 \pm 2.9$ & $\mathrm{f}$ & 0.0 & $54.68 \pm 3.0$ & de & 0.0 \\
\hline NINT- Anaerobic control & + & 3 & $0.0 \pm 0.0 \mathrm{a}$ & 100.0 & $0.0 \pm 0.0 \mathrm{a}$ & 100.0 & $0.0 \pm 0.0$ & a & 100.0 & $4.61 \pm 4.6$ & $\mathrm{a}$ & 100.0 \\
\hline NINT- Standard control & - & 3 & $0.0 \pm 0.0 \mathrm{a}$ & 100.0 & $0.0 \pm 0.0 \mathrm{a}$ & 100 & $0.0 \pm 0.0$ & $\mathrm{a}$ & 100.0 & $0.0 \pm 0.0$ & $\mathrm{a}$ & 100.0 \\
\hline Comp, $15 \mathrm{t} \mathrm{ha}^{-1}$ & + & 6 & $57.9 \pm 3.6 \mathrm{~d}-\mathrm{f}$ & 16.1 & $42.1 \pm 4.1 \mathrm{de}$ & 46.6 & $34.6 \pm 2.8$ & $\mathrm{~cd}$ & 41.8 & $38.23 \pm 4.0$ & b-d & 17.6 \\
\hline BCp, $15 \mathrm{t} \mathrm{ha}^{-1}$ & + & 6 & $49.4 \pm 3.9$ c-e & 28.4 & $36.6 \pm 5.0 \mathrm{~cd}$ & 53.6 & $35.1 \pm 5.4$ & c-e & 41.0 & $52.6 \pm 11.2$ & de & 100.0 \\
\hline WR, $15 \mathrm{t} \mathrm{ha}^{-1}$ & + & 6 & $60.3 \pm 3.1$ ef & 12.6 & $78.8 \pm 6.7 \mathrm{f}$ & 0.1 & $34.7 \pm 0.9$ & $c-e$ & 41.7 & $56.73 \pm 5.14$ & de & 49.9 \\
\hline $\mathrm{TM}, 2 \mathrm{gm}^{-2}$ & - & 6 & $7.8 \pm 4.7 \mathrm{ab}$ & 88.7 & $7.8 \pm 4.7 \mathrm{ab}$ & 90.1 & $15.7 \pm 1.6$ & $\mathrm{ab}$ & 73.6 & $17.89 \pm 6.3$ & $\mathrm{ab}$ & 84.6 \\
\hline INT- Anaerobic control & + & 6 & $58.2 \pm 4.5 \mathrm{~d}-\mathrm{f}$ & 15.7 & $61.4 \pm 4.3$ ef & 22.2 & $50.9 \pm 6.9$ & ef & 14.5 & $57.97 \pm 4.0$ & de & 14.0 \\
\hline INT- Standard control & - & 6 & $69.0 \pm 3.6 \mathrm{fg}$ & 0.0 & $78.9 \pm 3.9 \mathrm{f}$ & 0.0 & $59.5 \pm 3.4$ & $\mathrm{f}$ & 0.0 & $64.7 \pm 2.9$ & $\mathrm{e}$ & 0.0 \\
\hline NINT- Anaerobic control & + & 6 & $0.0 \pm 0.0$ a & 100.0 & $0.0 \pm 0.0 \mathrm{a}$ & 100.0 & $0.0 \pm 0.0$ & a & 100.0 & $0.0 \pm 0.0$ & $\mathrm{a}$ & 100.0 \\
\hline NINT- Standard control & - & 6 & $0.0 \pm 0.0 \mathrm{a}$ & 100.0 & $0.0 \pm 0.0 \mathrm{a}$ & 100.0 & $0.0 \pm 0.0$ & a & 100.0 & $0.0 \pm 0.0$ & a & 100.0 \\
\hline
\end{tabular}

a Comp: Compost; BCp: Brassica carinata pellet with anaerobic conditions; WR: Diplotaxis green manure; TM: Tolclofos methyl; INTAnaerobic control: Inoculated untreated control with anaerobic conditions; INT-Standard control: Inoculated untreated control without anaerobic conditions; NINT-Anaerobic control: Non-inoculated untreated control with anaerobic conditions; NINT-Standard control: Noninoculated untreated control without anaerobic conditions.

${ }^{\mathrm{b}}$ Means in each column accompanied by the same letter are not significantly different $(P \leq 0.05)$, according to Tukey's test. Standard errors are also indicated.

${ }^{c} \mathrm{E} \%$ : Disease reduction compared to the INT-Standard controls carried out for 3 or 6 weeks.

In the present study, effects of ASD using Brassicaceae species, either as wild rocket green manure or Brassica carinata dry pellets, and compost as C-sources, were evaluated on the lettuce- $R$. solani pathosystem, considering effects on basal rot incidence and on lettuce productivity (fresh weights) in ASD treatments simulated in growth chambers. Our aim was to test the different ASD $\mathrm{C}$-sources at one dosage equivalent to $15 \mathrm{t} \mathrm{ha}^{-1}$, with the only exception being the double dosage tested for WR green manure. The commercial dosage of $3 \mathrm{t} \mathrm{ha}^{-1}$ suggested for $B$. carinata pellets for biofumigation treatment was not considered. Our study was also carried out in two soil types, a sandy-loam alkaline soil and an acidic peat soil, at three temperatures $\left(21,26\right.$ or $\left.31^{\circ} \mathrm{C}\right)$, and with two treatment durations ( 3 or 6 weeks). Impacts of these treatments on lettuce grown in the treated and untreated soil was also assessed over two consecutive crop cycles carried out in greenhouse.

Carbon sources, temperatures, incubation periods and their interactions affected the efficacy of the ASD treatments. Differences in efficacy depended on the soil type. Rhizoctonia basal rot was reduced at the higher temperatures of $26^{\circ} \mathrm{C}$ or $31^{\circ} \mathrm{C}$ for 3 or 6 weeks in the peat soil, with resulting increased plant fresh weights, while generally in the sandy-loam soil, the greatest disease control was achieved with only 3 weeks of incubation, at all temperatures tested. In contrast, several studies have shown the impacts of temperature and ASD duration on pathogen survival, disease control and host yields in several pathosystems. Ebihara and Uematsu (2014) showed that Fusarium oxysporum f. sp. fragariae, Phytophthora cactorum and Verticillium dahliae, under anaerobic conditions, survived longer at $10^{\circ} \mathrm{C}$, and were eradicated more rapidly at $40^{\circ} \mathrm{C}$. Soil temperatures less than $30^{\circ} \mathrm{C}$ may be a critical factor in the effectiveness of ASD for reducing Fusarium wilt of strawberry (Muramoto et al., 2015; Shrestha et al., 2016).

Under the experimental conditions tested in the present study, different effects of the applied C-sources were observed. The impacts of Brassica crops and Brassicaceae seed meal have been shown to be effective biomasses for ASD treatments to control soil-borne diseases caused by Fusarium spp., Rhizoctonia spp., Pythium spp., and Verticillium spp. (Blok et al., 2000; Messiha et 
Table 7B. Mean percentages of lettuce plants affected by basal rot after application of different ASD soil treatments, carried out for 3 or 6 weeks, and at 21 or $31{ }^{\circ} \mathrm{C}$ in sandy-loam artificially infested with Rhizoctonia solani, at the end of the first and second crop cycles (Trial 2).

\begin{tabular}{|c|c|c|c|c|c|c|c|c|c|c|}
\hline \multirow{2}{*}{ Carbon source $^{\mathrm{a}}$, dosage } & \multirow{2}{*}{ ASD } & \multirow{2}{*}{$\begin{array}{c}\text { Treatment } \\
\text { duration } \\
\text { (weeks) }\end{array}$} & \multicolumn{4}{|c|}{ Cycle I } & \multicolumn{4}{|c|}{ Cycle II } \\
\hline & & & $21^{\circ} \mathrm{C}$ & $\mathrm{E} \%{ }^{\mathrm{c}}$ & $31^{\circ} \mathrm{C}$ & $\mathrm{E} \%$ & $21^{\circ} \mathrm{C}$ & E\% & $31^{\circ} \mathrm{C}$ & $\mathrm{E} \%$ \\
\hline Comp, $15 \mathrm{t} \mathrm{ha}^{-1}$ & + & 3 & $24.1 \pm 8.1 \quad b-d^{b}$ & 38.2 & $32.0 \pm 4.3 \mathrm{c}-\mathrm{e}$ & 27.8 & $15.4 \pm 2.5 \mathrm{a}-\mathrm{c}$ & 52.3 & $32.0 \pm 4.3 \mathrm{e}-\mathrm{g}$ & 22.7 \\
\hline BCp, $15 \mathrm{t} \mathrm{ha}^{-1}$ & + & 3 & $11.1 \pm 3.9 \mathrm{ab}$ & 71.5 & $19.7 \pm 4.2 b-d$ & 55.5 & $11.1 \pm 3.9 \mathrm{a}-\mathrm{c}$ & 65.6 & $9.0 \pm 3.1 \mathrm{a}-\mathrm{c}$ & 78.2 \\
\hline WR, $15 \mathrm{t} \mathrm{ha}^{-1}$ & + & 3 & $26.4 \pm 2.5 \mathrm{~b}-\mathrm{d}$ & 32.3 & $23.8 \pm 1.0 \mathrm{~b}-\mathrm{d}$ & 46.3 & $21.8 \pm 4.3$ b-e & 32.5 & $22.8 \pm 0.0 \mathrm{c}-\mathrm{f}$ & 44.9 \\
\hline $\mathrm{WR}, 30 \mathrm{t} \mathrm{ha}^{-1}$ & + & 3 & $24.0 \pm 4.2 \mathrm{~b}-\mathrm{d}$ & 38.5 & $18.1 \pm 2.0 \mathrm{bc}$ & 59.1 & $19.5 \pm 1.1$ be & 39.6 & $15.7 \pm 1.6$ a-d & 62.1 \\
\hline $\mathrm{TM}, 2 \mathrm{gm}^{-2}$ & - & 3 & $15.4 \pm 2.5 \mathrm{a}-\mathrm{c}$ & 60.5 & $11.5 \pm 7.8 \mathrm{ab}$ & 74.0 & $9.7 \pm 3.2 \mathrm{ab}$ & 70.0 & $11.5 \pm 7.8 \mathrm{a}-\mathrm{c}$ & 72.2 \\
\hline $\begin{array}{l}\text { BCp, } 15 \text { t ha-1- No anaerobic } \\
\text { control }\end{array}$ & - & 3 & $21.8 \pm 4.3$ b-d & 44.1 & $30.6 \pm 3.0 \quad c-e$ & 30.9 & $26.4 \pm 2.5 c-f$ & 18.3 & $9.7 \pm 3.2$ a-c & 76.6 \\
\hline INT- Anaerobic control & + & 3 & $32.1 \pm 3.7 \mathrm{~cd}$ & 17.7 & $35.5 \pm 0.8 \mathrm{de}$ & 19.9 & $32.1 \pm 3.7$ ef & 0.6 & $30.6 \pm 3.0 \mathrm{~d}-\mathrm{g}$ & 26.1 \\
\hline INT- Standard control & - & 3 & $39.0 \pm 4.7 \mathrm{~d}$ & 0.0 & $44.3 \pm 2.5 \mathrm{e}$ & 0.0 & $32.3 \pm 2.3$ ef & 0.0 & $41.4 \pm 1.4 \mathrm{f}$ & 0.0 \\
\hline NINT- Anaerobic control & + & 3 & $0.0 \pm 0.0 \mathrm{a}$ & 100.0 & $0.0 \pm 0.0 \mathrm{a}$ & 100.0 & $0.0 \pm 0.0 \mathrm{a}$ & 100.0 & $0.0 \pm 0.0 \mathrm{a}$ & 100.0 \\
\hline Comp, $15 \mathrm{t} \mathrm{ha}^{-1}$ & + & 6 & $32.3 \pm 2.3 \mathrm{~cd}$ & 1.5 & $34.7 \pm 2.0$ ce & 17.6 & $31.5 \pm 2.1$ ef & 19.2 & $35.5 \pm 0.8$ e-g & 15.7 \\
\hline BCp, $15 \mathrm{t} \mathrm{ha}^{-1}$ & + & 6 & $17.6 \pm 8.2$ a-c & 46.3 & $0.0 \pm 0.0$ a & 100.0 & $11.1 \pm 3.9 \mathrm{a}-\mathrm{c}$ & 72.5 & $0.0 \pm 0.0 \mathrm{a}$ & 100.0 \\
\hline WR, $15 \mathrm{t} \mathrm{ha}^{-1}$ & + & 6 & $15.7 \pm 1.6$ a-c & 52.1 & $21.1 \pm 3.4$ b-d & 49.9 & $15.7 \pm 1.6$ a-d & 59.7 & $20.2 \pm 2.9$ b-e & 52.0 \\
\hline WR, $30 \mathrm{tha}^{-1}$ & + & 6 & $17.7 \pm 3.2 \mathrm{a}-\mathrm{c}$ & 46.0 & $30.1 \pm 4.7 \mathrm{ce}$ & 28.5 & $17.7 \pm 3.2$ b-e & 54.6 & $16.5 \pm 2.7$ a-d & 60.8 \\
\hline $\mathrm{TM}, 2 \mathrm{gm}^{-2}$ & - & 6 & $11.1 \pm 3.9 \mathrm{ab}$ & 66.2 & $6.5 \pm 3.7 \mathrm{ab}$ & 84.6 & $11.1 \pm 3.9 \mathrm{a}-\mathrm{c}$ & 71.5 & $20.5 \pm 2.1$ b-e & 51.3 \\
\hline $\begin{array}{l}\text { BCp, } 15 \text { tha-1- No anaerobic } \\
\text { control }\end{array}$ & - & 6 & $28.9 \pm 2.1$ b-d & 13.1 & $12.2 \pm 4.7 \mathrm{ab}$ & 71.0 & $32.8 \pm 4.6$ ef & 15.9 & $27.3 \pm 2.6 c-f$ & 35.2 \\
\hline INT- Anaerobic control & + & 6 & $31.5 \pm 2.1$ b-d & 4.0 & $36.2 \pm 2.2 \mathrm{de}$ & 14.0 & $31.5 \pm 2.1$ ef & 19.2 & $36.2 \pm 3.0 \mathrm{fg}$ & 14.0 \\
\hline INT- Standard control & - & 6 & $32.8 \pm 4.6 \mathrm{~cd}$ & 0.0 & $42.1 \pm 3.2 \mathrm{e}$ & 0.0 & $39 \pm 4.7 \mathrm{f}$ & 0.0 & $42.1 \pm 3.2 \mathrm{~g}$ & 0.0 \\
\hline NINT- Anaerobic control & + & 6 & $0.0 \pm 0.0 \mathrm{a}$ & 100.0 & $0.0 \pm 0.0 \mathrm{a}$ & 100.0 & $0.0 \pm 0.0 \mathrm{a}$ & 100.0 & $0.0 \pm 0.0$ a & 100.0 \\
\hline
\end{tabular}

a Comp: Compost; BCp: Brassica carinata pellet with or without anaerobic conditions; WR: Diplotaxis green manure; TM: Tolclofos methyl; INT-Anaerobic control: Inoculated untreated control with anaerobiotic conditions; INT-Standard control: Inoculated untreated control without anaerobic conditions; NINT-Anaerobic control: Non inoculated untreated control with anaerobic conditions.

${ }^{\mathrm{b}}$ Means in each column accompanied by a common letter are not significantly different $(P \leq 0.05)$, according to Tukey's test. Standard errors are also reported.

${ }^{c} \mathrm{E} \%$ : Disease reduction compared to the INT-Standard controls carried out for 3 or 6 weeks.

al., 2007; Postma et al., 2014; Hewavitharan and Mazzola, 2016; Mowlick et al., 2012; 2013; Shennan et al., 2014; 2018). In the present study, Brassicaceae seed meal without anaerobic condition reduced basal rot of lettuce but to a lesser extent than in the ASD treatments, which generally provided more consistent disease control for all the tested temperatures in both soils (between a range of 52 to $72 \%$ disease reduction). However, the tested rate of application was five times greater than that suggested for the 'Biofence' product (3 $\mathrm{t} \mathrm{ha}^{-1}$ ) for biofumigation treatment. The economic value of such a high rate application should be evaluated. However, Butler et al., (2014 b) suggested that C source rates greater than $4 \mathrm{mg} \mathrm{g}^{-1}$ of soil were required when soil temperatures during ASD treatments were low (15$25^{\circ} \mathrm{C}$ ). Serrano-Pérez et al. (2017) confirmed that ASD under early spring conditions in Spain, using several C sources at $4 \mathrm{mg} \mathrm{g}^{-1}$ of soil, was effective to control $P$. nicotianae disease in pepper. Nevertheless, in the present study effects on disease reduction and lettuce fresh weights were not observed at the greatest WR green manure amount of $30 \mathrm{t} \mathrm{ha}^{-1}$. The efficacy of ASD basedWR was generally greatest at the highest incubation temperature in the peat soil and at the lowest temperature of $21^{\circ} \mathrm{C}$ in sandy loam soil. MaCarty et al. (2014) showed that an ASD treatment based on a mixture of Sinapis alba and Eruca sativa, affected survival of $R$. solani at approx. $20^{\circ} \mathrm{C}$, under accumulated anaerobic conditions of approx. $20,000 \mathrm{mVh}$. The accumulated soil anaerobic condition achieved in the present study during different treatments only partially explain the ASD efficacy. For instance, the greatest basal rot control was obtained from the Brassicaceae seed meal applied for 6 weeks in the sandy-loam soil at $31^{\circ} \mathrm{C}$, and this treatment resulted in $88,954 \mathrm{mVh}$. Almost the same value of cumulative $\mathrm{mVh}$, achieved at $21^{\circ} \mathrm{C}$, was only partially effective in the control of Rhizoctonia basal rot (28-46\% disease reduction), compared to the untreated controls. These were severely affected at the end of the first cultivation cycle. Also, the greatest efficacy of ASD using 
Table 8A. Mean fresh weight $\left(\mathrm{g} \mathrm{pot}^{-1}\right)$ of lettuce plants after application of different ASD soil treatments, carried out for 3 or 6 weeks, and at 21 or $26{ }^{\circ} \mathrm{C}$ in sandy-loam artificially infested with Rhizoctonia solani, at the end of the first and second crop cycles (Trial 1 ).

\begin{tabular}{|c|c|c|c|c|c|c|c|c|c|c|}
\hline \multirow{3}{*}{$\begin{array}{l}\text { Carbon source }{ }^{\mathrm{a}} \text {, dosage } \\
\text { Comp, } 15 \mathrm{t} \mathrm{ha}^{-1}\end{array}$} & \multirow{3}{*}{$\begin{array}{l}\text { ASD } \\
+\end{array}$} & \multirow{3}{*}{$\begin{array}{c}\begin{array}{c}\text { Treatment } \\
\text { duration } \\
\text { (weeks) }\end{array} \\
3\end{array}$} & \multicolumn{4}{|c|}{ Cycle I } & \multicolumn{4}{|c|}{ Cycle II } \\
\hline & & & \multicolumn{2}{|l|}{$21^{\circ} \mathrm{C}$} & \multicolumn{2}{|l|}{$26^{\circ} \mathrm{C}$} & \multicolumn{2}{|l|}{$21^{\circ} \mathrm{C}$} & \multicolumn{2}{|l|}{$26^{\circ} \mathrm{C}$} \\
\hline & & & $47.8 \pm 1.7$ & $\mathrm{de}^{\mathrm{b}}$ & $20.8 \pm 1.9$ & ef & $194.9 \pm 6.6$ & $\mathrm{bc}^{\mathrm{a}}$ & $166.1 \pm 34.4$ & $a-c$ \\
\hline BCp, $15 \mathrm{t} \mathrm{ha}^{-1}$ & + & 3 & $18.1 \pm 0.3$ & ef & $74.1 \pm 9.7$ & $b-d$ & $109.9 \pm 9.0$ & de & $110.7 \pm 20.5$ & $a-d$ \\
\hline WR, $15 \mathrm{t} \mathrm{ha}^{-1}$ & + & 3 & $16.0 \pm 1.5$ & ef & $7.4 \pm 1.3$ & f & $303.5 \pm 5.4$ & $\mathrm{a}$ & $67.4 \pm 19.4$ & $\mathrm{~d}$ \\
\hline $\mathrm{TM}, 2 \mathrm{gm}^{-2}$ & - & 3 & $67.2 \pm 6.6$ & $\mathrm{~cd}$ & $110.4 \pm 5.4$ & $a-c$ & $206.1 \pm 6.2$ & $\mathrm{bc}$ & $167.2 \pm 29.2$ & $a-c$ \\
\hline INT- Anaerobic control & + & 3 & $24.1 \pm 1.6$ & ef & $9.9 \pm 1.4$ & $\mathrm{f}$ & $170.9 \pm 18.5$ & $b-d$ & $74.5 \pm 15.9$ & $\mathrm{~cd}$ \\
\hline INT- Standard control & - & 3 & $13.3 \pm 2.8$ & $\mathrm{f}$ & $7.9 \pm 4.3$ & $\mathrm{f}$ & $78.8 \pm 9.7$ & $\mathrm{e}$ & $68.2 \pm 12.4$ & $\mathrm{~cd}$ \\
\hline NINT- Anaerobic control & + & 3 & $117.5 \pm 12.4$ & $\mathrm{ab}$ & $123.6 \pm 22.1$ & $a-c$ & $158.3 \pm 12.6$ & b-e & $194.8 \pm 27.3$ & $a-c$ \\
\hline NINT- Standard control & - & 3 & $105.2 \pm 2.3$ & $\mathrm{ab}$ & $88.2 \pm 3.9$ & $\mathrm{~cd}$ & $300.8 \pm 15.6$ & $\mathrm{a}$ & $204.9 \pm 38.5$ & $\mathrm{ab}$ \\
\hline Comp, $15 \mathrm{t} \mathrm{ha}^{-1}$ & + & 6 & $14.7 \pm 2.5$ & $\mathrm{f}$ & $13.8 \pm 0.6$ & $\mathrm{f}$ & $170.2 \pm 27.1$ & $b-d$ & $151.5 \pm 16.6$ & $a-c$ \\
\hline BCp, $15 \mathrm{t} \mathrm{ha}^{-1}$ & + & 6 & $5.6 \pm 0.9$ & $\mathrm{f}$ & $84.9 \pm 18.2$ & $\mathrm{~cd}$ & $236.2 \pm 24.3$ & $\mathrm{ab}$ & $94.7 \pm 32.5$ & $b-c$ \\
\hline $\mathrm{WR}, 15 \mathrm{t} \mathrm{ha}^{-1}$ & + & 6 & $25.4 \pm 6.9$ & ef & $4.8 \pm 2.8$ & $\mathrm{f}$ & $136.2 \pm 19.0$ & $c-e$ & $83.8 \pm 23.6$ & $b-d$ \\
\hline $\mathrm{TM}, 2 \mathrm{gm}^{-2}$ & - & 6 & $87.9 \pm 10.5$ & $a-c$ & $155 \pm 11.7$ & a & $152.2 \pm 14.3$ & $c-e$ & $203.6 \pm 24.9$ & $\mathrm{ab}$ \\
\hline INT- Anaerobic control & + & 6 & $2.5 \pm 1.1$ & $\mathrm{f}$ & $18.5 \pm 2.2$ & $\mathrm{f}$ & $88.2 \pm 19.6$ & $\mathrm{e}$ & $101.8 \pm 27.3$ & $a-d$ \\
\hline INT- Standard control & - & 6 & $17.6 \pm 9.5$ & ef & $5.4 \pm 2.3$ & $\mathrm{f}$ & $97.5 \pm 4.5$ & de & $57.2 \pm 17.0$ & $\mathrm{~d}$ \\
\hline NINT- Anaerobic control & + & 6 & $85.7 \pm 4.5$ & $\mathrm{bc}$ & $137.8 \pm 6.8$ & $\mathrm{ab}$ & $210.6 \pm 22.3$ & $\mathrm{bc}$ & $168.5 \pm 12.9$ & $a-c$ \\
\hline NINT- Standard control & - & 6 & $119.9 \pm 12.1$ & $\mathrm{a}$ & $103.6 \pm 6.6$ & $\mathrm{bc}$ & $200.1 \pm 13.2$ & $\mathrm{bc}$ & $223.5 \pm 26.0$ & $\mathrm{a}$ \\
\hline
\end{tabular}

a Comp: Compost; BCp: Brassica carinata pellet with anaerobic conditions; WR: Diplotaxis green manure; TM: Tolclofos methyl; INTAnaerobic control: Inoculated untreated control with anaerobic conditions; INT-Standard control: Inoculated untreated control without anaerobic conditions; NINT-Anaerobic control: Non-inoculated untreated control with anaerobic conditions; NINT-Standard control: Noninoculated untreated control without anaerobic conditions.

${ }^{\mathrm{b}}$ Means in each column accompanied by the same letter are not significantly different $(P \leq 0.05)$, according to Tukey's test. Standard errors are also indicated.

compost observed in the peat soil at $26^{\circ} \mathrm{C}$ for 6 weeks of incubation (67\% efficacy) resulted in $46,495 \mathrm{mVh}$, while, at the higher cumulative value of $95,550 \mathrm{mVh}$, achieved using compost as $\mathrm{C}$ - source at $31^{\circ} \mathrm{C}$, control of the disease was partial ( $20 \%$ disease reduction). Results from the present study are generally in agreement with those of Shennan et al. (2014; 2018), who provided evidence that accumulated soil anaerobic conditions of 50,000 $\mathrm{mV} \mathrm{h}^{-1}$ at $25^{\circ} \mathrm{C}$, achieved using wheat bran in a sandy clay loam soil, was crucial for control Verticillium dahliae in strawberry plants, but the same anaerobic condition did not provide efficient inactivation of the pathogen at $15^{\circ} \mathrm{C}$.

ASD efficacy is modulated by a complex mechanism, and since a low soil oxygen levels are prerequisites for pathogen inactivation (Runia et al., 2014) for some pathogens such as $R$. solani, efficacy is closely related to the carbon source. This is possibly due to different volatile profiles resulting from ASD treated soil, and to microbiological changes (MaCarty et al., 2014; Hewavitharana et al., 2014; 2015). Several studies have also reported that inoculum inactivation in soil under different temperature and accumulated soil anaerobic conditions during
ASD is pathogen specific. Mowlick et al., (2012; 2013) showed that the incubation temperature influenced the suppression of F. oxysporum f. sp. spinaciae to a great extent by stimulating the multiplication of the anaerobic bacteria related to the purely anaerobic clostridial groups in Brassica, oat and wheat bran ASD-treatments. The diversity in the clostridial groups was generally greatest in the Brassica and wheat bran ASD samples at $30^{\circ} \mathrm{C}$, and the diversity was greatly reduced at $20^{\circ} \mathrm{C}$ in the Brassica-treated soil.

The present study has shown that ASD based on Brassicaceae and compost as C-sources applied in a sandy-loam soil, had impacts on the control of $R$. sola$n i$ on lettuce, even at lower temperatures than those required for soil solarisation or biosolarisation (Gamliel, 2000), with a general improvement in disease control in the second crop cycle. For instance, the ASD based on compost was partially effective in reducing basal rot of lettuce at the end of the first crop cycle in both soils, while the greatest disease reductions of $53 \%$ and $66 \%$ were observed in the second crop cycle in the sandy-loam soil previously treated for 3 weeks at $21^{\circ} \mathrm{C}$. The benefits of long-term compost treatments on differ- 
Table 8B. Mean fresh weight $\left(\mathrm{g} \mathrm{pot}^{-1}\right)$ of lettuce plants after application of different ASD soil treatments, carried out for 3 or 6 weeks, and at 21 or $31{ }^{\circ} \mathrm{C}$ in sandy-loam artificially infested with Rhizoctonia solani, at the end of the first and second crop cycles (Trial 2).

\begin{tabular}{|c|c|c|c|c|c|c|c|c|c|c|}
\hline \multirow{3}{*}{$\begin{array}{l}\text { Carbon source }^{\mathrm{a}} \text {, dosage } \\
\text { Comp, } 15 \mathrm{t} \mathrm{ha}^{-1}\end{array}$} & \multirow{3}{*}{$\begin{array}{c}\text { ASD } \\
++\end{array}$} & \multirow{3}{*}{$\begin{array}{c}\begin{array}{c}\text { Treatment } \\
\text { duration } \\
\text { (weeks) }\end{array} \\
3\end{array}$} & \multicolumn{4}{|c|}{ Cycle I } & \multicolumn{4}{|c|}{ Cycle II } \\
\hline & & & \multicolumn{2}{|l|}{$21^{\circ} \mathrm{C}$} & \multicolumn{2}{|c|}{$31^{\circ} \mathrm{C}$} & \multicolumn{2}{|l|}{$21^{\circ} \mathrm{C}$} & \multicolumn{2}{|l|}{$31^{\circ} \mathrm{C}$} \\
\hline & & & $23.7 \pm 2.5$ & $\mathrm{~cd}^{\mathrm{b}}$ & $28.1 \pm 4.3$ & d-f & $71.8 \pm 7.4$ & $a-d$ & $26.9 \pm 7.7$ & fg \\
\hline BCp, $15 \mathrm{t} \mathrm{ha}^{-1}$ & + & 3 & $77.9 \pm 3.9$ & $\mathrm{~b}$ & $37.9 \pm 4.7$ & $\mathrm{~cd}$ & $94.7 \pm 9.0$ & $\mathrm{ab}$ & $149.5 \pm 18.2$ & $\mathrm{a}$ \\
\hline WR, $15 \mathrm{t} \mathrm{ha}^{-1}$ & + & 3 & $25.9 \pm 8.1$ & $\mathrm{~cd}$ & $26.3 \pm 1.0$ & $d-f$ & $77.9 \pm 6.4$ & $a-c$ & $56.0 \pm 5.0$ & $d-f$ \\
\hline $\mathrm{WR}, 30 \mathrm{t} \mathrm{ha}^{-1}$ & + & 3 & $26.0 \pm 4.2$ & $\mathrm{~cd}$ & $31.9 \pm 2.0$ & ce & $89.0 \pm 5.8$ & $a-c$ & $74.0 \pm 5.6$ & c-e \\
\hline $\mathrm{TM}, 2 \mathrm{gm}^{-2}$ & - & 3 & $38.2 \pm 2.3$ & $c$ & $43.6 \pm 3.7$ & $c$ & $64.1 \pm 12.4$ & $a-e$ & $64.3 \pm 20.1$ & $c-f$ \\
\hline BCp, $15 \mathrm{t} \mathrm{ha}^{-1}$ - No anaerobic control & - & 3 & $34.6 \pm 2.5$ & $\mathrm{~cd}$ & $30.4 \pm 4.2$ & ce & $96.1 \pm 23.2$ & $\mathrm{ab}$ & $77.8 \pm 8.1$ & b-e \\
\hline INT- Anaerobic control & + & 3 & $17.9 \pm 3.7$ & $\mathrm{~cd}$ & $19.4 \pm 3.0$ & $d-f$ & $60.1 \pm 5.9$ & a-e & $36.3 \pm 4.1$ & $d-f$ \\
\hline INT- No anaerobic control & - & 3 & $17.7 \pm 2.3$ & $\mathrm{~cd}$ & $15.3 \pm 2.0$ & ef & $48.1 \pm 12.8$ & b-e & $23.6 \pm 3.2$ & g \\
\hline NINT- Anaerobic control & + & 3 & $116.3 \pm 8.3$ & a & $109.8 \pm 5.6$ & $\mathrm{~b}$ & $92.2 \pm 8.4$ & $\mathrm{ab}$ & $127.1 \pm 7.1$ & $\mathrm{ab}$ \\
\hline Comp, $15 \mathrm{t} \mathrm{ha}^{-1}$ & + & 6 & $28.5 \pm 3.9$ & $\mathrm{~cd}$ & $15.7 \pm 2.5$ & ef & $51.7 \pm 5.0$ & b-e & $39.2 \pm 3.5$ & $d-f$ \\
\hline BCp, $15 \mathrm{t} \mathrm{ha}^{-1}$ & + & 6 & $67.3 \pm 3.6$ & $\mathrm{~b}$ & $50.0 \pm 0.0$ & $c$ & $83.4 \pm 8.7$ & $a-c$ & $150.4 \pm 12.9$ & a \\
\hline WR, $15 \mathrm{t} \mathrm{ha}^{-1}$ & + & 6 & $34.4 \pm 1.6$ & $\mathrm{~cd}$ & $28.9 \pm 3.4$ & $d-f$ & $56.1 \pm 6.2$ & b-e & $76.5 \pm 9.5$ & c-e \\
\hline WR, $30 \mathrm{t} \mathrm{ha}^{-1}$ & + & 6 & $22.3 \pm 3.2$ & $\mathrm{~cd}$ & $19.9 \pm 4.7$ & $d-f$ & $99.3 \pm 2.1$ & $\mathrm{ab}$ & $109.0 \pm 9.0$ & $a-c$ \\
\hline $\mathrm{TM}, 2 \mathrm{gm}^{-2}$ & - & 6 & $39.0 \pm 3.9$ & c & $34.5 \pm 0.8$ & $\mathrm{~cd}$ & $36.5 \pm 8.2$ & c-e & $77.1 \pm 9.3$ & b-e \\
\hline BCp, $15 \mathrm{t} \mathrm{ha}^{-1}$ - No anaerobic control & - & 6 & $32.4 \pm 8.2$ & $\mathrm{~cd}$ & $38.5 \pm 7.8$ & $\mathrm{~cd}$ & $111.6 \pm 22.1$ & a & $49.4 \pm 5.6$ & $d-f$ \\
\hline INT- Anaerobic control & + & 6 & $18.5 \pm 2.1$ & $\mathrm{~cd}$ & $13.8 \pm 2.2$ & $e-f$ & $22.8 \pm 1.6$ & de & $35.8 \pm 11.7$ & $d-f$ \\
\hline INT- No anaerobic control & - & 6 & $13.5 \pm 2.9$ & $\mathrm{~d}$ & $8.0 \pm 3.2$ & $\mathrm{f}$ & $14.3 \pm 3.6$ & e & $49.0 \pm 4.4$ & $d-f$ \\
\hline NINT- Anaerobic control & + & 6 & $85.3 \pm 6.7$ & $\mathrm{~b}$ & $141.5 \pm 6.1$ & a & $69.0 \pm 3.2$ & $a-d$ & $158.4 \pm 7.5$ & $\mathrm{a}$ \\
\hline
\end{tabular}

${ }^{a}$ Comp: Compost; BCp: Brassica carinata pellet with or without anaerobic conditions; WR: Diplotaxis green manure; TM: Tolclofos methyl; INT-Anaerobic control: Inoculated untreated control with anaerobiotic conditions; INT-Standard control: Inoculated untreated control without anaerobic conditions; NINT-Anaerobic control: Non inoculated untreated control with anaerobic conditions.

${ }^{\mathrm{b}}$ Means in each column accompanied by a common letter are not significantly different $(P \leq 0.05)$, according to Tukey's test. Standard errors are also reported.

ent pathosystems are well known (Abawi and Widmer, 2000; Chellemi, 2002; Gamliel, 2000; Lazarovits and Subbarao, 2010). However, a wide range of results have been achieved when compost has been used as organic amendments, including decreases and increases in soilborne disease incidence and severity (Hoitink and Fahy, 1986; Abbasi et al., 2002; Noble and Coventry, 2005; Bonanomi et al., 2007; Pugliese et al., 2011; 2015). Compost suppressiveness against Rhizoctonia solani in particular is known to be limited (Termorshuizen et al., 2006; Pugliese et al., 2015), and the use of composted manure for ASD has also been less effective than other carbon sources for controlling this pathogen (Hewavitharana and Mazzola, 2016).

Generally, the variation in efficacy of individual treatment combinations was limited in the second crop cycle, with positive effect on lettuce yields. However, the infection of plants by Rhizoctonia solani was generally less in the second crop cycle than in the first, and all the tested C-sources applied to both soils provided greater disease control in the second crop cycle, which resulted in increased lettuce yields.
Although it is well known that ASD treatments may have effects on soil fertility, by influencing different soil properties (MaCarty et al., 2014; Butler et al., 2014b; Di Gioia et al., 2017), they may also have negative effects on crop yields, due to low amounts of available $\mathrm{N}$ when large amounts of labile C are applied (Whitmore, 1996), or to phytotoxicity. Phytotoxic effects of ASD treatments could explain the low lettuce fresh weights measured after the first cycle, which may not only be attributable to differences in efficacy of the tested ASD treatments. High-N amendments in soils can lead to the production of ammonia, which is toxic to a wide range of pathogens and nematodes (Conn et al., 2005; López-Robles et al., 2013; Mazzola et al., 2018), but also may have negative effects on plant development (Barker et al., 1970). Readily decomposable amendments, under anaerobic conditions, can also favour the production of toxic organic acids which have been linked to the control of fungal, nematode, insect, and weed pests (Okazaki and Nose, 1986; Conn et al., 2005; Momma et al., 2006; Hestmark et al., 2019). The possible phytotoxicity to lettuce from organic acid accumulation or salts during ASD treatments should be considered. 
In the present study small increases in soil $\mathrm{pH}$ of 0.2 to 0.4 units was observed when $B$. carinata pellets were used. Increased suppressiveness of Rhizoctonia damping-off of sugar beet in a near neutral to alkaline soil, compared to acid soil, from use of dried peanut plant residues, has been reported, due to increased activity of specific antagonistic soil microorganisms (Watanabe et al., 2011). Similar disease suppression effects have been obtained by amending soils with composts rich in cellulolytic and oligotrophic actinomycete antagonists (Tuitert et al., 1998; Kasuya et al., 2006; Ros et al., 2006). Changes in the soil microbiome, induced by an ASD treatment in a carbon source dependent manner (Hewavitharan and Mazzola, 2016), can be persistent, and may result in long-term pathogen re-infestation (Goud et al., 2004). Improved disease suppression and yields were observed for the ASD treatment-based compost in the second crop cycle, which is similar to other reports (Ros et al., 2006; Hestmark et al., 2019). Nevertheless, the increased lettuce fresh weights observed after ASD treatment in the second crop cycle may also be explained in part by improved nutritional status of the plants, as was observed by Butler et al., (2014 b) and Di Gioia et al., (2017).

No chemical or non-chemical methods used alone exhibit the same efficacy as some soil fumigants used in the past (Katan, 2017), so disease reduction offered by ASD studied here is promising. The potential of ASD for controlling $R$. solani incidence in lettuce merits further investigation, so that it can be adapted to different local conditions. The significant but only partial efficacy of ASD when Brassica carinata was applied as C-source does not justify the adoption of such treatment in the practice, but this approach integrated with other disease management methods could be worthwhile (Butler et al., 2012 a). For sustainable agriculture, the use of compost or Diplotaxis green manure as other C-sources possible for evaluation of other green wastes. ASD based on Diplotaxis could be useful for managing crop residues in horticultural systems for ready-to-eat salad crops, where stringent quality requirements must be satisfied and crop damage thresholds of $5 \%$ make the products unmarketable.

\section{ACKNOWLEDGEMENTS}

This study received funding from the European Union's Horizon 2020 research and innovation programme EUCLID EU-CHINA Lever for IPM Demonstration, Grant agreement no. 633999. The authors thank Stefano Demarchi for technical support and Marguerite
Jones for English language revision and Prof. Jaacov Katan and the anonymous referees for their valuable suggestions.

\section{CONFLICT OF INTEREST}

Massimo Pugliese declares he has a financial interest (shareholder) in AgriNewTech, the company that provided the compost tested in this research.

\section{LITERATURE CITED}

Abawi G. S., Widmer T. L., 2000. Impact of soil health management practices on soilborne pathogens, nematodes and root diseases of vegetable crops. Applied Soil Ecology 15: 37-47.

Abbasi P.A., Al-Dhmani J., Sahin F., Hoitink H.A.J. Miller S.A., 2002. Effect of compost amendments on disease severity and yield of tomato in conventional and organic production systems. Plant Disease 86:156161.

Barker A.V., Maynard D.N., Mioduchowska B., Buch A., 1970. Ammonium and salt inhibition of some physiological processes associated with seed germination. Physiol. Plant 23: 898-907.

Barrière V., Lecompte F., Nicot F.C., Maisonneuve B., Tchamitchian M., Lescourret F., 2014. Lettuce cropping with less pesticides. Agronomy for Sustainable Agriculture. A review 34 : 175-198.

Blancard D., Lot H., Maisonneuve B., 2003. Maladies des salades: identifier, connaitre et maîtriser. INRA, Paris.

Blok W.J., Lamers J.G., Termorshuizen A.J., Bollen G.J., 2000. Control of soilborne plant pathogens by incorporating fresh organic amendments followed by tarping. Phytopathology 90: 253-259.

Bonanomi G., Antignani V., Pane C., Scala F., 2007. Suppression of soilborne fungal diseases with organic amendments. Journal of Plant Pathology 89: 311-324.

Butler D. M., Kokalis-Burelle N., Muramoto J., Shennan C., McCollum T. G., Rosskopf E. N. 2012a. Impact of anaerobic soil disinfestation combined with soil solarization on plant parasitic nematodes and introduced inoculum of soilborne plant pathogens in raised-bed vegetable production. Crop Protection 39: 33-40.

Butler D. M., Rosskopf E. N., Kokalis-Burelle N., Albano J. P., Muramoto J., ... Shennan C., 2012b. Exploring warm season cover crops as carbon sources for anaerobic soil disinfestation (ASD). Plant and Soil 355: $149-165$. 
Butler D.M., Ownley B.H., Dee M.E., Inwood S.E., McCarty D.G., Rosskopf E.N., 2014a. Low carbon Amendment rates during anaerobic soil disinfestation (ASD) at moderate soil temperatures do not decrease viability of Sclerotinia sclerotiorum sclerotia or Fusarium root rot of common bean. Acta Horticulturae 1044: 19-28.

Butler D.M., Kokalis-Burelle N., Albano J.P., McCollum T.G., Muramoto J., ... Rosskopf E.N., 2014 b. Anaerobic soil disinfestation (ASD) combined with soil solarization as a methyl bromide alternative: vegetable crop performance and soil nutrient dynamics. Plant Soil 378: 365-381.

Chellemi D., 2002. Nonchemical management of soilborne pests in fresh market vegetable production systems. Phytopathology 92:1367-1372.

Conn K.L., Tenuta M., Lazarovits G., 2005. Liquid swin e manure can kill Verticillium dahliae microsclerotia in soil by volatile fatty acid, nitrous acid, and ammonia toxicity. Phytopathology 95: 28-35.

Di Gioia F., Ozores-Hamptona M., Zhaob X., Thomasc J., Wilsonc P., Rosskopf E., 2017. Anaerobic soil disinfestation impact on soil nutrients dynamics and nitrous oxide emissions in fresh-market tomato. Agriculture, Ecosystems and Environment 240: 194-205.

Ebihara Y., Uematsu S., 2014. Survival of strawberrypathogenic fungi Fusarium oxysporum f. sp. fragariae, Phytophthora cactorum and Verticillium dahliae under anaerobic conditions. Journal General Plant Pathology 80: 50-58.

Fiedler S., Vepraskas M.J., Richardson J.L., 2007. Soil Redox Potential: Importance, Field Measurements, and Observations. Advances in Agronomy 94:1-54.

Gamliel A., 2000. Soil amendments: a non chemical approach to the management of soilborne pests. Acta Horticulturae 532: 39-47.

Garibaldi A., Gilardi G., Gullino M.L., 2014. Critical aspects in disease management as a consequence of the evolution of soil-borne pathogens. Acta Horticulturae 1044: 43-52.

Goud J.K.C., Termorshuizen A.J., Blok W.J., Van Bruggen A.H.C., 2004. Long-term effect of biological soil disinfestation on Verticillium wilt. Plant Disease 88: 688-694.

Gullino M.L., Gilardi G., Garibaldi A., 2019. Ready-to-eat salad crops: the plant pathogen's heaven. Plant Disease 103:2153-2170.

Hestmark K.V., Fernández-Bayo J.D., Harrold D.R., Randall T.E., Achmon Y., Vander Gheynst J.S., 2019. Compost induces the accumulation of biopesticidal organic acids during soil biosolarization. Resources Conservation Recyclcling 143: 27-35.
Hewavitharana S. S., Mazzola M., 2016. Carbon sourcedependent effects of anaerobic soil disinfestation on soil microbiome and suppression of Rhizoctonia solani AG-5 and Pratylenchus penetrans. Phytopathology 106: 1015-1028.

Hewavitharana S.S., Ruddell D., Mazzola M., 2014. Carbon source-dependent antifungal and nematicidal volatiles derived during anaerobic soil disinfestation. European Journal of Plant Pathology 140: 39-52.

Hewavitharana S. S., Shennan C., Muramoto J., Mazzola M., 2015. Anaerobic soil disinfestation disease control performance in strawberry as influenced by environmental variables. Phytopathology 105: S4.59.

Hoitink H.A.J., Fahy P.C., 1986. Basis for the control of soilborne plant pathogens with composts. Annual Review of Phytopathology 24: 93-114.

Huang X., Liu L., Wen T., Zhang J., Wang F., Cai F., 2016. Changes in the soil microbial community after reductive soil disinfestation and cucumber seedling cultivation. Applied Microbiology Biotechnology 100: 5581-5593.

Kasuya M., Olivier A.R., Ota Y., Tojo M., Honjo H., Fukui R., 2006. Induction of soil suppressiveness against Rhizoctonia solani by incorporation of dried plant residues into soil. Phytopathology 96: 13721379.

Katan J., 2017. Diseases caused by soilborne pathogens: biology, management and challenges. Journal of Plant Pathology 99: 305-315

Katase M, Kubo C, Ushio S, Ootsuka E, Takeuchi T, Mizukubo T., 2009. Nematicidal activity of volatile fatty acids generated from wheat bran in reductive soil disinfestation. Japanese Journal of Nematology 39: 53-62.

Lamers J.G., Runia W.T., Molendijk L.P.G., Bleeker P.O., 2010. Perspectives of anaerobic soil disinfestation. Acta Horticulturae 883: 277-283.

Lazarovits G., Subbarao K., 2010. Challenges in controlling Verticillium wilt by the use of non chemical methods. In: Recent developments in Management of Plant Diseases, Plant Pathology in the 21stCentury (Gisi U., Chet I., Gullino M.L. Eds.), Springer, Dordrecht, NL, 247-264.

López-Robles J., Olalla C., Rad C., Díez-Rojo M.A., López-Pérez J.A., ... Rodríguez-Kábana R., 2013. The use of liquid swine manure for the control of potato cyst nematode through soil disinfestation in laboratory conditions. Crop Protection 49: 1-7.

Mazzola M., Brown J., Izzo A.D., Cohen M.F., 2007. Mechanism of action and efficacy of seed mealinduced pathogen suppression differ in a Brassicaceae species and time-dependent manner. Phytopathology 97: 454-460. 
Mazzola M., Granatstein D.M., Elfving D.C., Mullinix K., 2001. Suppression of specific apple root by Brassica napus seed meal amendment regardless of glucosinolate content. Phytopathology 91: 673-679.

Mazzola M., Hewavitharana S., 2014. Carbon sourcedependent volatile production and ASD efficacy for suppression of apple root pathogens and parasites. Acta Horticulturae 1044: 208-214.

Mazzola M., Manici L.M., 2012. Apple replant disease: role of microbial ecology in cause and control. Annual Review of Phytopathology 50: 45-65.

Mazzola M., Muramoto J., Shennan C., 2018. Anaerobic disinfestation induced changes to the soil microbiome, disease incidence and strawberry fruit yields in California field trials. Applied Soil Ecology 127: 74-86.

McCarty D. G., Inwood S. E. E, Ownley B. H., Sams C. E., Wszelaki A. L., Butler, D. M., 2014. Field evaluation of carbon sources for anaerobic soil disinfestation in tomato and bell pepper production in tennessee. HortScience 49: 272-280.

Messiha N.A.S., van Diepeningen A.D., Wenneker M., van Beuningen A.R., Janse J.D., ...Blok W.J., 2007. Biological Soil Disinfestation (BSD), a new control method for potato brown rot, caused by Ralstonia solanacearum race 3 biovar 2. European Journal of Plant Pathology 117: 403-415.

Momma N., Yamamoto K., Simandi P., Shishido M., 2006. Role of organic acids in the mechanisms of biological soil disinfestation (BSD) Journal General Plant Pathology 72: 247-252.

Momma N., Momma M., Kobara Y., 2010. Biological soil disinfestation using ethanol: Effect on Fusarium oxysporum f. sp. lycopersici and soil microorganisms. Journal of General Plant Pathology 76: 336-344.

Momma N., Kobara Y., Uematsu S., Kita N., Shinmura A., 2013. Development of biological soil disinfestations in Japan. Applied Microbiology Biotechnology 97: 3801-3809.

Mowlick S., Takehara T., Kaku N., Ueki K., Ueki A., 2012. Proliferation of diversified clostridial species during biological soil disinfestation incorporated with plant biomass under various conditions. Applied Microbiology Biotechnology 97: 8365-8379.

Mowlick S., Yasukawa H., Inoue T., Takehara T., Kaku N., ..., Ueki A., 2013. Suppression of spinach wilt disease by biological soil disinfestation incorporated with Brassica juncea plants in association with changes in soil bacterial communities. Crop Protection 54: 185193.

Muramoto J., Shennan C., Zavatta M., Yoyama L., Hewavitharana S.S., Mazzola M., 2015. Controlling
Fusarium wilt of strawberries by anaerobic soil disinfestation. Pages 16.1-4. Proc. Annu. Int. Res. Conf. Methyl Bromide Altern. Emiss. Reduct. MBAO, San Diego, CA.

Noble R., Coventry R., 2005. Suppression of soil-borne plant diseases with composts: a review. Biocontrol Science and Technology 15: 3-20.

Okazaki H., Nose K., 1986. Acetic acid and N-butyric acid as causal agents of fungicidal activity of glucoseamended flooded soil. Ann Phytopathol Soc Jpn 52: 384-393.

Postma J., Schilder M.T., Stevens L.H., 2014. Potential of Organic Amendments to Enhance Soil Suppressiveness against Rhizoctonia solani Disease in Different Soils and Crops. Acta Horticolturae 1044:127-132.

Pugliese M., Liu B.P., Gullino M.L., Garibaldi A., 2011. Microbial enrichment of compost with biological control agents to enhance suppressiveness to soilborne diseases. Journal of Plant Disease and Protection 118: 45-50.

Pugliese M., Gilardi G., Garibaldi A., Gullino M. L., 2015. Organic Amendments and Soil Suppressiveness: Results with Vegetable and Ornamental Crops. In: Organic Amendments and Soil Suppressiveness in Plant Disease Management (Meghvansi M. K. and Varma A. eds.), Soil Biology 46, Springer, ISBN 9783-319-23074-0, 495-509.

Rabenhorst M.C., Castenson K.L., 2005. Temperature effects on iron reduction in a hydric soil. Soil Science 170: 734-742.

Rodríguez-Molina M.C., Serrano-Pérez P., Palo C., 2016. Effect of biofumigation with brassica pellets combined with Brassicaceae cover crops and plastic cover on the survival and infectivity of inoculum of Phytophthora nicotianae Breda de Haan. Pest Managagement Science 72: 1295-1301.

Ros M., Klammer S., Knapp B., Aichberger K., Insam H., 2006. Long-term effects of compost amendment of soil on functional and structural diversity and microbial activity. Soil Use Manage 22: 209-218.

Rosskopf E.N., Serrano-Pérez P., Hong J., Shrestha U., Rodríguez-Molina M.C., ... Butler D., 2015. Anaerobic soil disinfestation and soilborne pest management. Soil Biology 46: 277-305.

Runia W.T., Molendijk L.P.G., Ludeking D.J.W., Schomaker C.H., 2012. Improvement of anaerobic soil disinfestation. Comm. Appl. Biol. Science 77: 753-762.

Runia W.T., Thoden T.C., Molendijk L.P.G., van den Berg W., Termorshuizen A.J., ..., Meints H., 2014. Unravelling the mechanism of pathogen inactivation during anaerobic soil disinfestation. Acta Horticulturae 1044: 177-193. 
Serrano-Pérez P., Rosskopf E., De Santiago A., RodríguezMolina M.C., 2017. Anaerobic soil disinfestation reduces survival and infectivity of Phytophthora nicotianae chlamydospores in pepper. Scientia Horticolturae 215: 38-48

Shennan C., Muramoto J., Koike S., Bolda M., Daugovish O., 2010. Optimizing anaerobic soil disinfestation for non-fumigated strawberry production in California. HortScience 45: S270.

Shennan C., Muramoto J., Lamers J., Mazzola M., Rosskopf E.N., ..., Kobara Y., 2014. Anaerobic soil disinfestation for soil borne disease control in strawberry and vegetable systems: current knowledge and future directions. Acta Horticulturae 1044: 157-165.

Shennan C., Muramoto J., Koike S., Baird G., Fennimore S., ...Mazzola M., 2018. Anaerobic soil disinfestation is an alternative to soil fumigation for control of some soilborne pathogens in strawberry production. Plant Pathology 67: 51-66.

Shinmura A., 2004. Principle and effect of soil sterilization methods by reducing the redox potential of soil. PSJ Soilborne Disease Workshop Report 22: 2-12.

Shrestha U., Auge R., Butler D., 2016. A meta-analysis of the impact of anaerobic soildisinfestation on pest suppression and yield of horticultural crops. Frontiers Plant Science 7: doi.org/10.3389/fpls.2016.01254.

Sneh B., Jabaji-Hare S., Neate S., Dijs G., 1996. Rhizoctonia Species: Taxonomy, Molecular Biology, Ecology, Pathology and Disease Control. Dordrecht, Netherlands, Kluwer Academic, San Francisco.

Strauss S. L., Kluepfel D.A., 2015. Anaerobic soil disinfestation: A chemical-independent approach to preplant control of plant pathogens. Journal of Integrative Agriculture 14: 2309-2318.

Tamietti G., Garibaldi A., 1987. Effectiveness of soil solarization against Rhizoctonia solani in northern Italy. Proc CEC/IOBC Group Meeting on Integrated Pest Management in Protected Vegetable Crops, 27-29 May 1987. Cabrils, Spain, pp 193-197.

Termorshuizen A. J., van Rijn E., van der Gaag D. J., Alabouvette C., Chen Y., ..., Zmora-Nahum S., 2006. Suppressiveness of 18 composts against 7 pathosystems: Variability in pathogen response. Soil Biology \& Biochemistry 38: 2461-2477.

Tuitert G., Szczech M., Bollen G.J., 1998. Suppression of Rhizoctonia solani in potting mixtures amended with compost made from organic household waste. Phytopathology 88:764-773.

Watanabe K., Matsui M., Honjo H., Becker J.O., Fuk R., 2011. Effects of soil pH on Rhizoctonia damping-off of sugar beet and disease suppression induced by soil amendment with crop residues. Plant Soil 347: 255268.
Whitmore A.P., 1996. Modelling the release and loss of nitrogen after vegetable crops. Net Journal Agricultural Science 44: 73-86.

Yossen V., Zumelza G., Gasoni L., Kobayashi K., 2008. Effect of soil reductive sterilization on Fusarium wilt in greenhouse carnation in Cordoba, Argentina. Australasian Plant Pathology 37: 520-522. 
